\title{
Stable and fair congestion control mechanisms for cooperative vehicle safety in vehicular networks
}

\author{
Neda Nasiriani \\ West Virginia University
}

Follow this and additional works at: https://researchrepository.wvu.edu/etd

\section{Recommended Citation}

Nasiriani, Neda, "Stable and fair congestion control mechanisms for cooperative vehicle safety in vehicular networks" (2013). Graduate Theses, Dissertations, and Problem Reports. 635.

https://researchrepository.wvu.edu/etd/635

This Thesis is protected by copyright and/or related rights. It has been brought to you by the The Research Repository @ WVU with permission from the rights-holder(s). You are free to use this Thesis in any way that is permitted by the copyright and related rights legislation that applies to your use. For other uses you must obtain permission from the rights-holder(s) directly, unless additional rights are indicated by a Creative Commons license in the record and/ or on the work itself. This Thesis has been accepted for inclusion in WVU Graduate Theses, Dissertations, and Problem Reports collection by an authorized administrator of The Research Repository @ WVU. For more information, please contact researchrepository@mail.wvu.edu. 
STABLE AND FAIR CONGESTION CONTROL MECHANISMS FOR COOPERATIVE VEHICLE SAFETY IN VEHICULAR NETWORKS

\author{
by \\ Neda Nasiriani \\ Thesis submitted to the \\ Benjamin M. Statler College of Engineering and Mineral Resources \\ at West Virginia University \\ in partial fulfillment of the requirements for the degree of \\ Master of Science \\ in \\ Electrical Engineering
}

Yaser P. Fallah, Ph.D., Chair

Parviz Famouri, Ph.D.,

Tim Menzies, Ph.D.

Lane Department of Computer Science and Electrical Engineering

Morgantown, West Virginia

2013

Keywords: Cooperative Vehicular Safety; Channel Busy Ratio; Congestion Control; Stability; Fairness; Power Control.

CCopyright 2013 Neda Nasiriani 


\begin{abstract}
STABLE AND FAIR CONGESTION CONTROL MECHANISMS FOR

COOPERATIVE VEHICLE SAFETY IN VEHICULAR NETWORKS
\end{abstract}

by Neda Nasiriani

Cooperative vehicle safety (CVS) systems operate based on broadcast of vehicle position and safety information to neighboring cars. The communication medium of CVS is a Dedicated Short Range Communication (DSRC) wireless channel. One of the main challenges in large scale deployment of CVS systems is the issue of scalability. To address the scalability problem, several congestion control methods have been proposed and are currently under field study. These mechanisms adapt transmission rate and power, based on network measures such as: Channel Busy Ratio (CBR), packet reception rate and vehicles tracking error. Channel Busy Ratio (CBR) is defined as the ratio of time that the channel is sensed busy during a time slot. We examine two such algorithms named Linear Memoryless Range Control (LMRC) and Gradient Descent Range Control (GRC). The dynamic behavior of these algorithms over time and space has been studied to evaluate temporal stability and spatial fairness. Moreover we have introduced a novel congestion control mechanism named Stateful Utilization-Based Power Adaptation (SUPRA) which is fundamentally a stateful version of LMRC algorithm to control power of transmission in contrast to Range Control algorithms. The control function of SUPRA has the ability to be tailored to different road conditions from low to high densities and different rates of transmission. The stability of the algorithm is proven through complete analysis of control function and verified in numerous simulation runs for typical road scenarios.Fairness is another issue which is probable to happen in different scenarios. An Unfair situation is when cars having the same density and rate of transmission, do not have analogous share of the channel. We have proposed a distributed mechanism to resolve the issue of unfairness. In this method each node sends out its sensed CBR along with safety messages to neighboring nodes, so each node will have access to all CBRs received from neighbors as well as its own measured CBR. This will provide a wide-ranging view of the whole channel and consequently adaptation of range/power of transmission will be done based on an aggregation of all these CBR values. 
To my beloved busband Ali and my family 


\section{ACKNOWLEDGMENTS}

First, I would like to thank my advisor, Dr. Yaser P. Fallah for his advice and provision throughout the course of my research. His innovative ideas and encouragement helped me to pursue my research successfully. This thesis would not be complete without his advice and support.

I greatly appreciate my committee members, Prof. Parviz Famouri and Prof. Tim Menzies for their time and support during my studies at WVU. Special thanks to Prof. Menzies for his encouragement and motivation. His love for learning and research has always been an inspiration to me. I greatly thank Prof. Famouri for his advice and support during my research. I thank him for the opportunities he made for me to show my capabilities in specially GTC project.

I wish to acknowledge the Lane Department of Computer Science and Electrical Engineering to give me the opportunity to pursue my Master in an educating, competitive and supportive environment.

I would like to thank my husband, Ali for his unconditional and endless love. He was greatly supportive and encouraging during last two years. At times of frustration when I was so tired and stressed out he was the one to give me hope, energy and motivation to stand up again and continue my path to success. He was the one who listened patiently to all my concerns and helped me to sort out anxieties and overcome them. 
I would like to sincerely thank my parents and sisters for their love, support and benevolence. I could not have achieved what I got today without my father inspiration and encouragement during my life. I am sure that my mother prayer has been with me all the time and I could not have achieved what I got today without her compassionate soul praying for me. I would like to thank my lovely sisters for their love specially Nasrin, who was motivating and encouraging me all the time. She helped me to get back together in times of doubt by positive energy and emotional support. 


\section{TABLE OF CONTENTS}

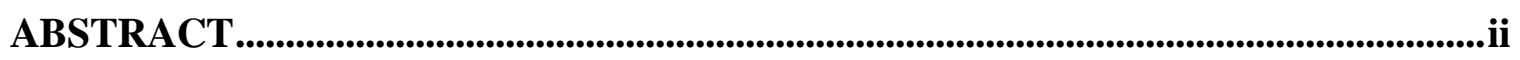

1 Introduction to Vehicular Ad hoc Wireless Networks..................................................9

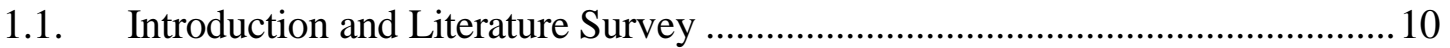

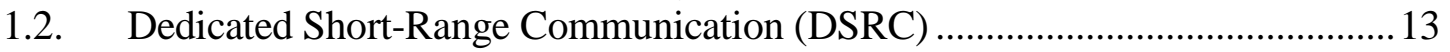

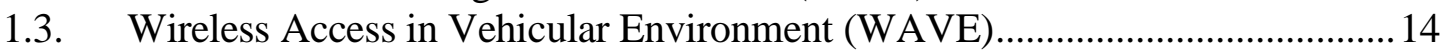

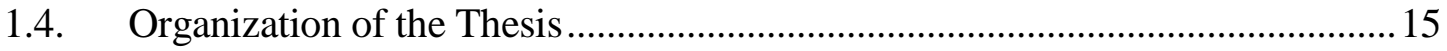

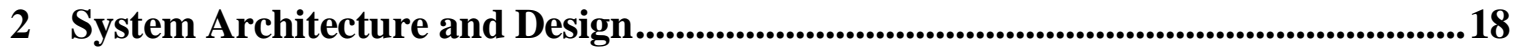

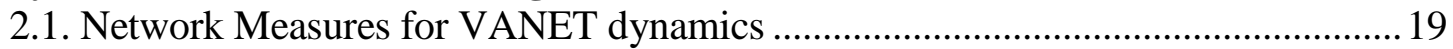

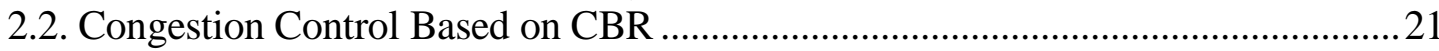

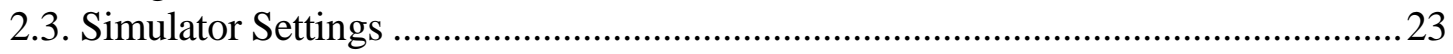

3 Range Control Algorithms .................................................................................................................26

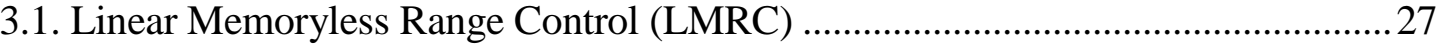

3.1.1. Stability Analysis of LMRC Algorithm .....................................................29

3.1.2. Study of LMRC for Static Highway Scenarios ............................................... 31

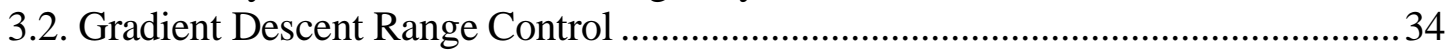

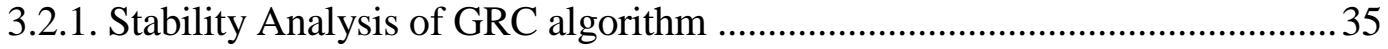

3.3. Study of Congestion Control Algorithms at Intersections ..................................... 37

3.3.1. Result of Linear Memoryless Range Control Algorithm in Intersection

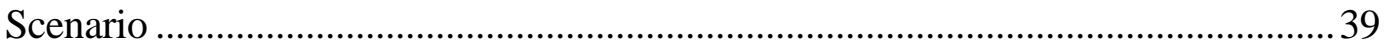

3.3.2. Result of Gradient Descent Range Control Algorithm in Intersection........... 41

3.4. Verification in More Realistic Scenarios for LMRC and GRC algorithms ............45

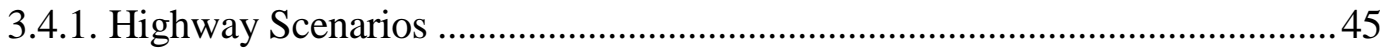

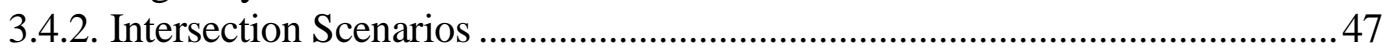

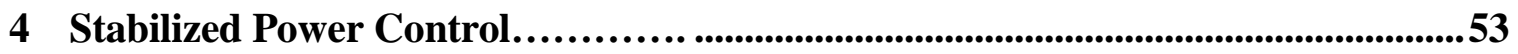

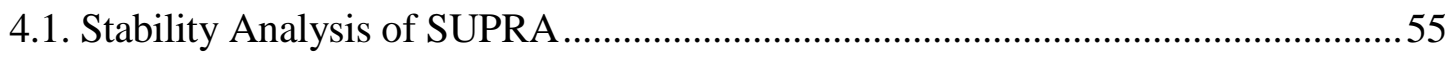

4.2. Result of SUPRA on Static and Realistic Scenarios ............................................6 63

5 Fairness Assurance for Power Control ..........................................................................6 67

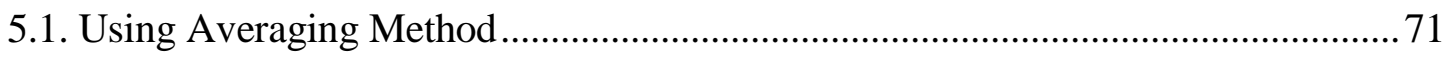

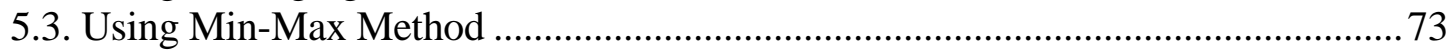

5.4. Analysis of CBR Enhancement Algorithm ........................................................ 74

6 Conclusion and Future Work ..............................................................................................................81 


\section{LIST OF FIGURES}

Figure 1-1 WAVE system dedicated frequency at 5.9 GHz band ............................................. 14

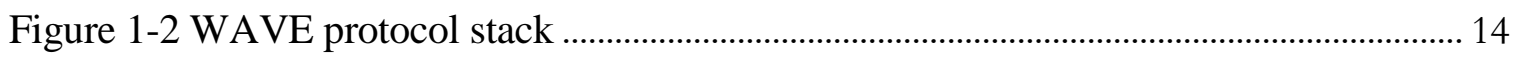

Figure 2-1 schematic of rate and range controller ............................................................................ 18

Figure 2-2 IDR vs. CBR for different values of rate(5-115 msg/sec), range(20-400m), and $\rho(0.1-0.2$ vehicle/m). Points belonging to the same experiment with different values of $d$ are connected by dotted line and different colors; although due to overlap they are indistinguishable. [13] 20

Figure 2-3 Information Dissemination Rate for different rate (1-15) $\mathrm{msg} / \mathrm{sec}$ and ranges of transmission(20-400)m 22

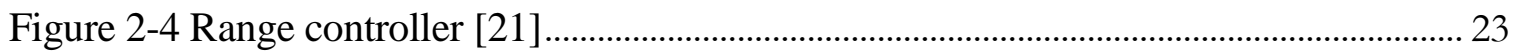

Figure 2-5 overview of Wi-Fi sub layers in NS3 ..................................................................... 24

Figure 2-6 distance bins for Nakagami fading model implemented in NS3 ............................ 25

Figure 3-1 Communication characteristic curves for six different scenarios, and feedback

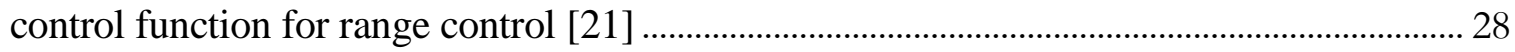

Figure 3-2 Divergence for LMRC algorithm for $D \min , D \max =(50,300), \ldots \ldots \ldots \ldots \ldots \ldots \ldots \ldots . . . . . . . . . . .30$

Figure 3-3 Convergence study on communication characteristic curve for $\rho=0.1$ and

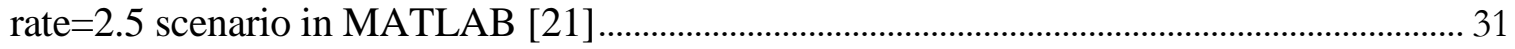

Figure 3-4 LMRC algorithm for $\rho=0.05$ and rate=5 scenario [21] ....................................... 32

Figure 3-5 LMRC algorithm result for a mixed scenario which has a $\rho=0.1$ up to 1500 meter of road and $\rho=0.02$ for the next half. [21]

Figure 3-6 LMRC algorithm for $\rho=0.05$ and rate=5 scenario with limits $($ Dmin, Dmax $)=(50,300)($ Umin, Umax $)=(0.4,0.8)$, the algorithm does not converge in time result showing the range chosen by each node. [21].

Figure 3-7 convergence steps for GRC algorithm for scenario with $\rho=0.05$ rate $=5$ and $\eta=50$ in MATLAB. [21]

Figure 3-8 Result of GRC algorithm with $\eta=50$ for case $\rho=0.05$ and rate $=5$, unfairness is observed in this case [23] 37

Figure 3-9 Snapshot at time $=30$ of LMRC Algorithm for mixed scenario with high density $(0.2-0.1-0.05)$ and rate $=5[23]$ 
Figure 3-10 Result of LMRC algorithm with parameters in (5) for case $\rho=0.2$ and rate $=5[23]$

Figure 3-11 Result of LMRC algorithm with parameters in (7) for mixed scenario with high density (0.2-0.1-0.05) and rate=5 [23]. 41

Figure 3-12 Result of GRC with $\eta=50$ for case $\rho=0.2$ and rate $=5$ [23] 42

Figure 3-13 Result of GRC algorithm with $\eta=50$ for mixed scenario with high density $(0.2-0.1-0.05)$ and rate $=5[23]$ 43

Figure 3-14 Result of GRC with $\eta=50$ for case $\rho=0.1$ and rate $=5$ [23] 43

Figure 3-15 Result of GRC algorithm with averaging $\eta=50$ for case $\rho=0.2$ and rate $=5[23]$ 44

Figure 3-16. Result of GRC algorithm with averaging, $\eta=50$ for mixed scenario with high density (0.2-0.1-0.05) and rate=5 [23]. 45

Figure 3-17 LMRC algorithm for free flow scenario (max speed= $70 \mathrm{Mph}$ ) [21] ................ 46

Figure 3-18 LMRC algorithm for low speed scenario (max speed= $30 \mathrm{Mph}$ ) [21] .............. 47

Figure 3-19 SUMO simulator GUI [23]

Figure 3-20 Result of GRC algorithm with averaging $\eta=100$ for SUMO low density in 2 ways scenario, East-West street, rate $=5$ [23]

Figure 3-21 Result of GRC algorithm with averaging $\eta=100$ for SUMO low density in 2 ways scenario, South-North street, rate $=5$ [23]

Figure 3-22 Result of GRC algorithm with averaging $\eta=100$ for SUMO high density in 4 ways scenario, East-West street, rate $=5$ [23] 50

Figure. 3-23 Result of GRC algorithm with averaging $\eta=100$ for SUMO high density in 4 ways scenario, South-North street, rate $=5$ [23] 51 Figure. 3-24 Result of LMRC for SUMO high density in 4 ways scenario East-West street, rate $=5[23]$ 52

Figure 4-1 SUPRA control schema 55

Figure 4-2 Communication characteristic curves for eight different scenarios, Power Control function (solid red line), Operational Area of the SUPRA-Control (dashed lined rectangle in blue) 56

Figure 4-3 schematic illustration of SUPRA control on a sample network characteristic curve approximated as a linear function. 57 
Figure 4-4 illustration of different states for two consecutive steps of control function ...... 57 Figure 4-5 matlab simulation for $\rho=0.04$ and rate=5 top) SUPRA divergence with $\eta=0.96$ middle) SUPRA convergence with $\eta=0.9$ Bottom) SUPRA fast convergence with $\eta=0.562$ Figure 4-6 SUPRA algorithm result $\eta=0.5$, for $\rho=0.05$ and rate $=5 \mathrm{~Hz}$.................................. 64 Figure 4-7 SUPRA algorithm, $\eta=0.5$, scenario $\rho=0.03$ and rate $=5 \mathrm{~Hz}$................................. 64 Figure 4-8 SUPRA algorithm result $\eta=0.5$, for realistic scenario (free flow) rate $=5 \mathrm{~Hz} \ldots . . .65$ Figure 4-9 SUPRA algorithm result $\eta=0.5$, for realistic scenario (low speed) rate $=5 \mathrm{~Hz} \ldots .66$ Figure 5-1.The edge nodes density ...... 69

Figure 5-2 GRC algorithm for scenario $\rho=0.05$ and rate $=5 \mathrm{~Hz}$ with gain $=50 \ldots \ldots \ldots \ldots \ldots \ldots . . . .70$ Figure 5-3 Result of GRC algorithm with averaging, $\eta=50$ for case $\rho=0.05$ and rate $=571$ Figure 5-4 Nodes q and $\mathrm{z}$ use lower power due to higher local CBR (without the enhanced algorithm) 75

Figure 5-5 In an unfair situation, false network characteristic curves $\mathrm{Hg}$ and $\mathrm{Lg}$ are observed by nodes seeing unbalanced high and low CBRs, resulting in false and unfair stability point (square); the real network characteristic curve $g$ and its stability point (circle) will result when nodes are synchronized using the enhanced algorithm. 76 Figure 5-6 SUPRA algorithm, $\eta=0.5$ and Enhancement applied on top of SUPRA, realistic scenario (low speed) rate $=5$. 78

Figure 5-7 SUPRA algorithm, $\eta=0.5$ and Enhancement applied on top of SUPRA, scenario $\rho=0.03$ and rate $=5 \mathrm{~Hz}$ 78 Figure 5-8 SUPRA algorithm, $\eta=0.5$ and Enhancement applied on top of SUPRA, scenario $\rho=0.04$ and rate $=5 \mathrm{~Hz}$ with unfairness build up 79 Figure 5-9 SUPRA algorithm, $\eta=0.5$ and No Enhancement, scenario $\rho=0.04$ and rate $=5 \mathrm{~Hz}$ with unfairness build up 80 


\section{LIST OF TABLES}

Table 2-1 Nakagami fading parameters for simulation setup ..................................................... 25

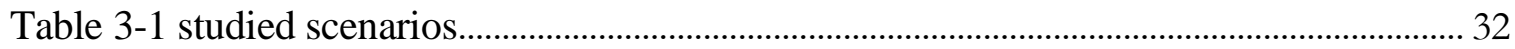

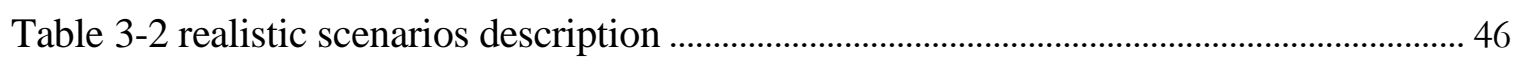

Table 4-1 gain $(\eta)$ values for typical scenarios, with $P \max =25 \mathrm{dBm}$ and $P \min =5 \mathrm{dBm}$,

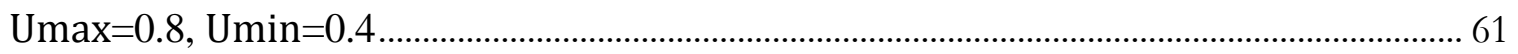

Table 5-1 Hypothetical unfairness resolved by using fairness enhancement.......................... 77 


\section{LIST OF ACRONYMS}

\begin{tabular}{|c|c|}
\hline Acronym & Definition \\
\hline $\mathrm{AC}$ & Access Category \\
\hline AIFS & Arbitrary Inter-Frame Space \\
\hline BSM & Basic Safety Message \\
\hline CSMA/CA & Career Sense Multiple Access/Collision Avoidance \\
\hline CVSS & Cooperative Vehicle Safety System \\
\hline CBR & Channel Busy Ratio \\
\hline $\mathrm{CCH}$ & Control Channel \\
\hline DSRC & Dedicated Short Range Communication \\
\hline EDCA & Enhanced Distributed Channel Access \\
\hline FCC & Federal Communication Commission \\
\hline GRC & Gradient Descent Range Control \\
\hline IDR & Information Dissemination Rate \\
\hline ITS & Intelligent Transportation System \\
\hline LMRC & Linear memoryless Range Control \\
\hline MAC & Medium Access Control \\
\hline NCC & Network Characteristic Curve \\
\hline OBU & On-Board Unit \\
\hline OFDM & Orthogonal Frequency- Division Multiplexing \\
\hline QoS & Quality of Service \\
\hline $\mathrm{SCH}$ & Service Channel \\
\hline SUPRA & Stateful Utilization-based PoweR Adaptation \\
\hline $\mathrm{V} 2 \mathrm{~V}$ & Vehicle to Vehicle \\
\hline V2I & Vehicle to Infrastructure \\
\hline VANET & Vehicular Ad-hoc NETwork \\
\hline WAVE & Wireless Access in Vehicular Environment \\
\hline
\end{tabular}




\section{Chapter 1 : INTRODUCTION TO VEHICULAR AD HOC WIRELESS NETWORKS}

Wireless communication has revolutionized human life by increasing awareness, information dissemination and safety. This type of communication is supposed to be very helpful in existing systems such as transportation system. Intelligent Transportation Systems (ITS) has emerged by adding communication, computation and control units to existing vehicles. The communication part is empowered by wireless communication which enables message transmission between cars. The addition of these units has significantly increased the safety and efficiency of legacy transportation systems. On-Board Units (OBU) equipped with IEEE 802.11p transceivers enable vehicle to vehicle (V2V) as well as Vehicle to Infrastructure communications (V2I). V2V communication is the foundation of Cooperative Vehicle Safety (CVS) systems. The CVS Systems operates based on vehicular ad-hoc network (VANET) which is meant to be a one-hop communication. Most of the safety applications work based on one-hop communication since neighboring nodes will mostly be involved in safety actions of each vehicle. In case of demand for more complex networking and multi-hop communication, these amenities should be managed in application layer rather than lower layers. 


\subsection{Introduction and Literature Survey}

Cooperative vehicle safety systems (CVSS) [1] [5] is one of the most important applications of Vehicular Ad-hoc Networks (VANETs). In CVSS, vehicles send out their position and safety information to their neighboring vehicles to have a map of surrounding vehicles and track their position and status. This system operates on Dedicated Short Range Communication (DSRC) and WAVE as its communication medium and rely on wireless communication for delivery of critical vehicle safety and tracking information. CVSS performance relies on delivery of these safety messages over the shared dedicated channel. Therefore, performance can be highly affected due to the limited capacity of the channel as well as considerable rate of interference due to hidden node effect. Consequently, there has been a lot of effort in literature to have an optimum controller for the network to assure a certain level of awareness.

The basic design of V2V communication in CVS systems used $10 \mathrm{~Hz}$ transmission of Basic Safety Messages (BSM) up to a distance of $250 \mathrm{~m}$. This design caused the issue of scalability and congestion in even very common and simple traffic scenarios. Therefore, a great deal of effort has been spent on finding a solution to the scalability issue in CVS systems. These works have been directed at the Congestion Control path, which tries to resolve congestion (reactive methods) or avoid it (proactive methods) by adjusting rate or/and range/power of transmission. One notable work which addresses the issue from a proactive approach is [17]; vehicles in this method adapt their power using a max-min approach, assuming a defined maximum load for the channel. Reactive Congestion Control mechanisms can be grouped into two main categories: 1) Application Specific 2) Network feedback Oriented. In some literature [15] the first type is considered as a subordinate to 
the second type of congestion control mechanisms; however here we will give a short description of both categories in literature. In application specific methods such as [1] the authors try to reduce load imposed by lower priority messages in favor of higher priority packets in a cooperative way. The priority is calculated based on speed of node, density of surrounding nodes, and level of importance of the application for each message. In [11] more application specific characteristics have been considered for a lane change assistance application, such as using the fact that if there are 2 vehicles obstructing the lane for the $3^{\text {rd }}$ vehicle to change its lane, the third vehicle just need one message from the closer vehicle and no more messages needed from the second vehicle; the authors also claim that in case of multiple applications requirements, adjusted powers can be merged.

In the second category, adaptation is based on feedback from the network to control levers such as rate and range/power of transmission. In [10] the authors use feedback from other nodes to see if they are covering the required range of transmission or not. This method could suffer from collided packets which would not be received and the feedback can be incomplete. [18] Huang et. al use a kinematic estimator to assess the perceived error at the receiver and based on this error and whether it is deviating from the error threshold or not, the node will decide to send out the packet. A limited feedback of the channel defined as Channel Busy Ratio (CBR) has been introduced by Fallah et. al. in [13]. The Information Dissemination Rate (IDR), which is a measure of channel throughput, has been shown to be a convex function of CBR. Therefore, the authors in [13] claim that maintaining CBR around 0.7 can maximize the IDR. Many studies [13][15][20][21] as well as this paper consider CBR as an appropriate feedback of the channel. In range control mechanism introduced in [13] authors use a sub-optimal method to maintain CBR in a range (0.4-0.8) to have maximum utilization of the channel in terms of throughput (IDR) while prevent to 
make the channel congested. A thorough analysis of temporal stability and spatial fairness of the work in [13] has been studied in [21][22] and will be expanded in the next chapter of this paper. Stability is shown to be guaranteed by a slight change in the algorithm while fairness is improved by augmenting an enhancement to the algorithm which uses neighboring nodes CBR in addition to locally measured CBR to broaden its view of the network. Although these two parameters (rate or range/power of transmission) can both contribute to the problem, in abovementioned efforts, the authors are trying to control the channel load by adapting one of rate or range/power of transmission. In works like [15][20] authors are trying to adjust both range and rate simultaneously. In [15] Tielert et. al. first adjust the range of transmission to maximize IDR and then the adaptation of rate using CBR is performed; on the other hand in [20] authors suggest an alternative and first adjust the rate of transmission based on error dependent policy and then perform the adaptation for Range. The latter method is being tested by industry through CAMP project and is under field study right now.

The contributions of this work are mainly as follows:

1) Studying stability analysis of existing algorithms which are being used by industry like LMRC and GRC. These algorithms have been studied thoroughly for different probable densities and rates of transmission.

2) Proposing a new stabilized direct power control named Steteful Utilization-based PoweR Adaptaion (SUPRA). Its stability analysis which is proved to be stable in time for all different road densities and transmission rates. This algorithm is designed to allow fast convergence as well as stability in time and fairness in space. 
3) Presenting a new mechanism named distributed measurements of CBR in contrast to local CBR calculations. Two different aggregation methods have been introduced for this system named: Averaging and Min-Max. Min-Max approach has been applied on top of SUPRA algorithm and enhanced fairness of the algorithm. The unfairness issue is completely resolved using this Min-Max enhancement on top of SUPRA algorithm. Therefore, a stable and fair congestion control mechanism has been introduced in this work which can be used by industry to help CVSS happening faster.

\subsection{Dedicated Short-Range Communication (DSRC)}

The US Federal Communications Commission (FCC) has dedicated $75 \mathrm{MHz}$ of band in 5.9 GHz spectrum. This band is reserved for Vehicular safety and application communications for Intelligent Transportation Systems (ITS) purposes. The layout of DSRC channel can be observed in Figure 1-1. The bandwidth of this channel is separated into eight sub-channels, of which six are named Service Channel $(\mathrm{SCH})$, one is reserved as Control Channel $(\mathrm{CCH})$ and one is guard channel. SCH is used for non-safety services while $\mathrm{CCH}$ is dedicated to safety applications; finally, the guard channel is reserved for guard services. This separation makes priority differentiation between safety and regular messages easier. The safety application messages have different priority levels which are applied at application layer but they all share the same channel 178 and their access to the shared channel is regulated by queuing mechanisms for different Access Categories (AC). 


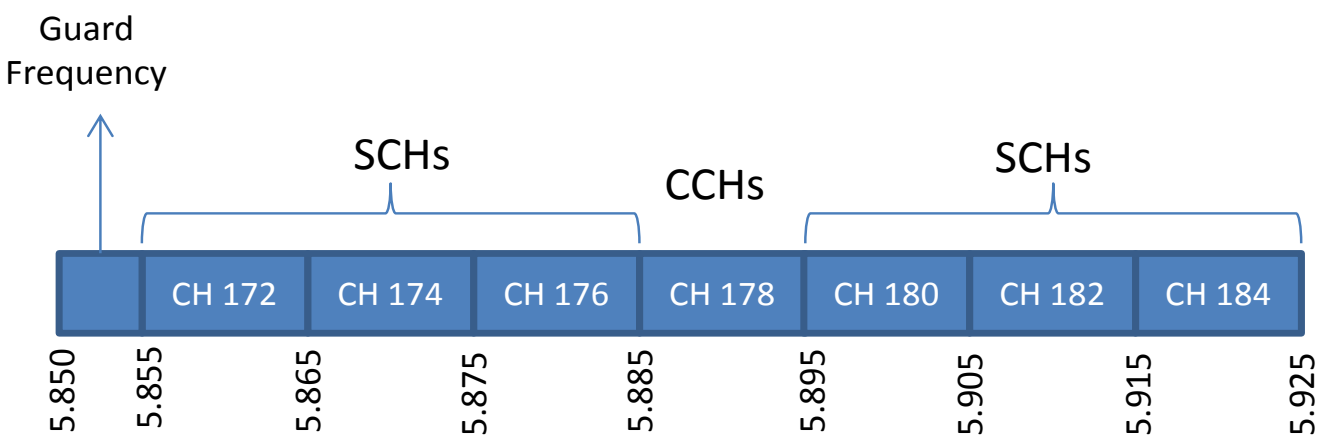

Figure 1-1 WAVE system dedicated frequency at $5.9 \mathrm{GHz}$ band

\subsection{Wireless Access in Vehicular Environment (WAVE)}

Wireless Access in Vehicular Environments (WAVE) standard [3] controls the wireless access mechanism to DSRC channel. This standard includes the IEEE 802.11p for physical and MAC layers and IEEE 1609.x for higher layers coordination such as security issues and multi-channel usage. IEEE 802.11p operates based on Orthogonal Frequency-Division Multiplexing (OFDM) technology in contrast to IEEE 802.11 standard [4][2][7][6]. The WAVE protocol stack schematic can be observed in Figure 1-2.

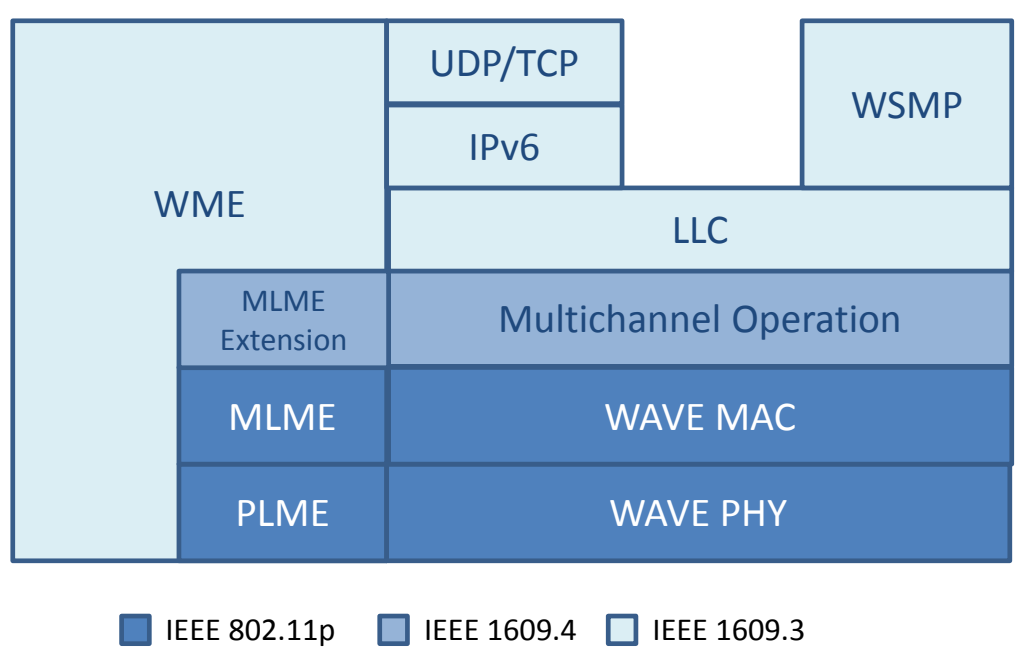

Figure 1-2 WAVE protocol stack 
The basic Medium Access Control (MAC) operation is standardized in IEEE 802.11p and follows the Distributed Coordination Function (DCF). DCF operates based on Carrier Sense Multiple Access with Collision Avoidance (CSMA/CA) mechanism to let the nodes access the channel. There is a MAC extension defined for WAVE which is used from Enhanced Distributed Channel Access (EDCA) of IEEE 802.11e protocol. EDCA adds Access Category (AC) which allows Quality of Service (QoS) objectives by prioritizing and an additional inter frame spacing named, Arbitrary Inter-Frame Space (AIFS). AIFS helps to guarantee the priorities by dedicating smaller wait time to packets queued in higher priority ACs.

\subsection{Organization of the Thesis}

The main contribution of this thesis is to study stability of existing congestion control mechanisms such as LMRC and GRC algorithms and finally propose a more robust version of LMRC named SUPRA to guarantee stable and fair congestion control throughout the network.

In chapter 2 a short description of system architecture for existing congestion control schemes is given. Two of the most common and effective congestion control mechanisms are controlling the rate or range/power of transmission. A schematic of these methods is presented in Figure 2-1. In the same chapter the most dominant feedback measure for congestion control is being introduced which is named Channel Busy Ratio(CBR). The logic behind using CBR as network feedback is introduced in details. For network simulations we use NS3 simulator and the setup for simulation scenarios such as 
propagation model, fading model, packet size and rate of transmission is explained in the last section of chapter2.

Chapter 3 is the most comprehensive chapter of this work. In this chapter we mostly introduce aforementioned congestion control mechanisms: LMRC and GRC. It starts with presenting the algorithms complete dynamics and then continues with stability analysis of the algorithms. A set of convergence conditions for LMRC is defined and a few improvements have been made to the algorithm to increase its performance, stability and fairness. The algorithms are applied in 2 different set of scenarios: 1) highway scenario 2) intersection scenario. Each scenario has been studied separately considering its unique characteristics. Afterward a set of realistic simulations scenarios have been used to verify the algorithms performance and solidness in case of moving vehicles imitating real situations.

Chapter 4 contains the largest contribution of this work which is introducing an improved, stateful version of LMRC algorithm titled Stateful Utilization-Based PoweR Adaptation(SUPRA) algorithm. The stability analysis of this algorithm has been studied next in the same chapter and convergence condition is presented as well. The robustness and stability of this algorithm is shown through extensive simulation results as well as realistic scenario set ups.

The other side issue of congestion control which was observed in our simulation studies was fairness. There are some situations in which the channel is not shared fairly between all the vehicles which are having the same density and rate of transmission, this issue is named unfairness. Chapter 5 addresses the issue of unfairness and suggests two mechanisms on 
top of congestion control mechanisms to guarantee fairness. Finally the result of SUPRA with using the fairness enhancement is presented which shows great stability and robustness for most of the cases. This result assures that using SUPRA with the fairness enhancement can guarantee stability and fairness for all the vehicles without having LMRC limitations. 


\section{Chapter 2 : SYSTEM ARCHITECTURE AND DESIGN}

As explained in previous part in CVS systems we have 2 tunable parameters (Range and Rate of Transmission) and according to the literature there are a few controller designs for each of rate and range control mechanisms such as [13][15][18][19][20]. The schematic of the general design for these controllers can be observed in Figure 2-1.

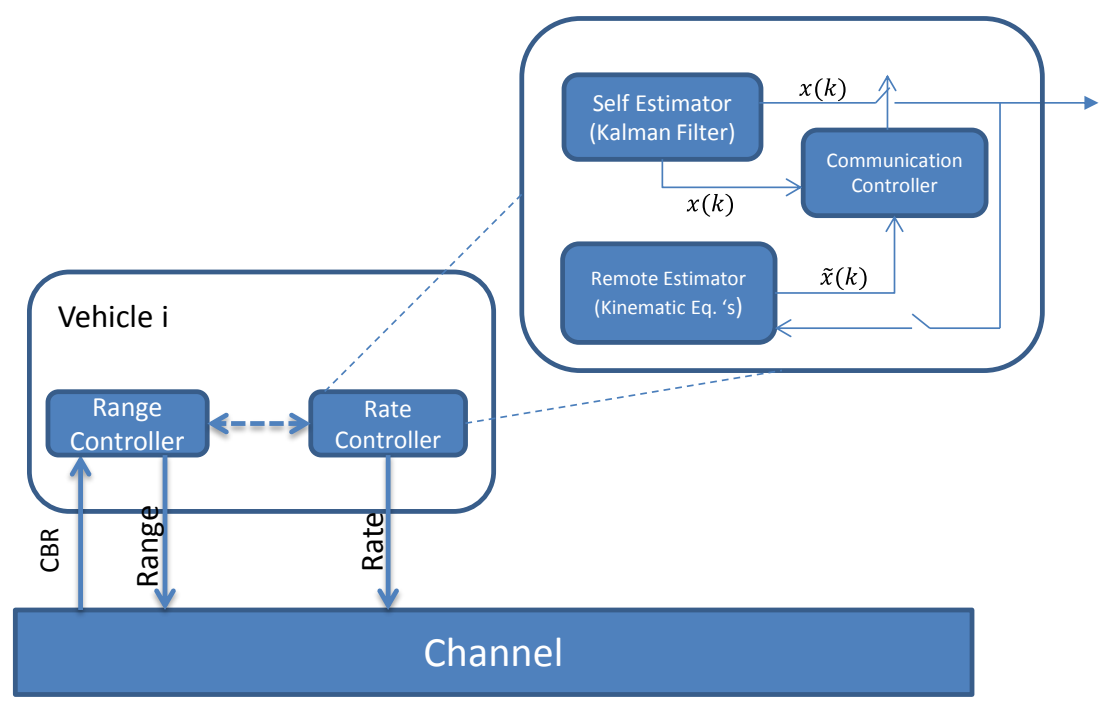

Figure 2-1 schematic of rate and range controller

We will study the range/power control in this letter and rate control is not in the scope of this study. However reasonable assumptions about average rate are made throughout this work which represents the average rate of transmission in different scenarios based on previous studies [20] which is $5 \mathrm{~Hz}$ and $2.5 \mathrm{~Hz}$. In next part a few measurements of VANET has been introduced and defined. These measurements are used in this study to characterize 
the dynamics of the system and design the controller for the system based in these dynamics; therefore a comprehensive introduction to them is important.

\subsection{Network Measures for VANET dynamics}

In order to control congestion in the channel in the most efficient way we have to examine different controllable parameters of CVSS. In CVSS a few parameters such as rate and range of transmission have the highest contribution to congestion build up. However there are other parameters such as contention window size which can affect the channel utilization but this effect is more limited and therefore not studied in this work. The next step is to quantify the effect of rate and range of transmission on the system to be able to design a controller. The modeling of the whole system is believed to be intractable so far because of complexities caused by hidden node and CSMA/CA characteristics of the MAC layer. Therefore, we are considering a performance measure named IDR which is in line with CVSS performance to guarantee maximum awareness up to 400 meters. IDR considers rate of reception at receivers, which directly affects the level of awareness and number of nodes which receive the packets in certain vicinity; in a nut shell, IDR considers range of coverage as well as frequency of updated values at receiver up to a certain distance. Information Dissemination Rate (IDR) is defined as the number of copies of a packet delivered per unit time from a single vehicle to its neighbors up to a given distance $d_{\text {max }}$; this measure mainly gives a sense of throughput of the channel.

$$
I D R=\sum_{i \in \text { Setof Neighborsuptod }_{\max }} r . P_{\text {suc }}(i)
$$


In [13] the authors studied IDR, versus channel utilization named Channel Busy Ratio (CBR), for different rate and range of transmission and different road densities. In the same study as shown in Figure 2-2 the authors claim that IDR for all different parameters settings fall on the same dome shaped graph, independent of rate, range of transmission and density of nodes in the network. CBR or channel Utilization (u) is defined as network congestion metric which is easily available at each node. The local value of CBR can be computed by a vehicle using clear channel assessment (CCA) reports from the physical layer to MAC layer in 802.11. Averaging the CCA reading in time, a node can calculate the local CBR. $\mathrm{CBR}$ is considered as a limited feedback measure which shows the performance of the network.

$$
u=\left(\sum_{i=1}^{\left[T / T_{\text {slot }}\right]} \Lambda_{i}\right) /\left[T / T_{\text {slot }}\right]
$$

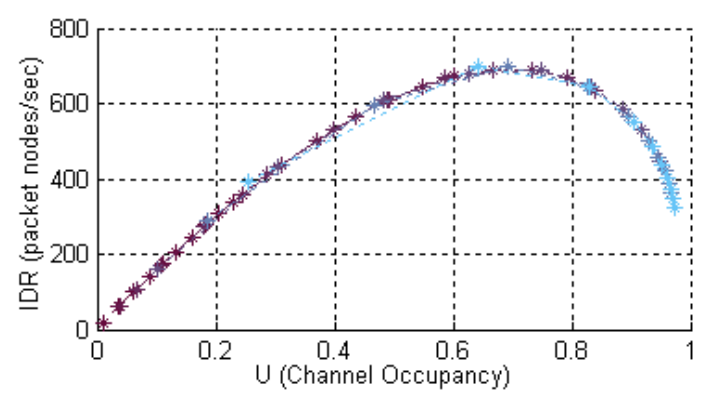

Figure 2-2 IDR vs. CBR for different values of rate(5-115 msg/sec), range(20$400 \mathrm{~m})$, and $\rho(0.1-0.2$ vehicle/m). Points belonging to the same experiment with different values of $\mathrm{d}$ are connected by dotted line and different colors; although due to overlap they are indistinguishable. [13]

Network Characteristics Curve:

In order to make analytic studies of algorithms less intricate, we have exercised Network Characteristic Curve (NCC) which is a statistical model of the network for a certain density and rate of transmission. In CVSS, total throughput of the channel is a function of number 
of nodes and their rate and range of transmission; therefore, by fixing number of nodes and transmission rate we can capture the behavior of range assignments in the channel on IDR and subsequently CBR. This behavior is shown in terms of NCCs which gives the CBR and is a function of Range. A set of NCCs for typical road densities can be observed in Figure 2-4 in solid blue line. NCC is shown as $U_{k}=g\left(D_{k}\right)$, for stability analysis. These graphs have been extracted from extensive simulation runs in NS3. For each scenario we ran the simulation for 20 seconds for each range assignment (overall $10 * 20=200 \mathrm{sec}$ ). Consequently NCC as a statistical model of each scenario will ease the testing of algorithms stability and this is the approach that is used through this work. However after complete analysis, the algorithms are further verified using NS3 simulation runs for both static and realistic scenarios. The result can be observed in following sections.

\subsection{Congestion Control Based on CBR}

The observation in [13] imply that channel performance in terms of IDR is solely a function of CBR and is independent of other parameters in CVSS such as rate, range of transmission and number of nodes(density). This claim is supported by the modeling work in [22] as well; in latter study the shape of the network performance is correlated to the type of protocol which is CSMA/CA with hidden node effect. This sets the stage for introducing CBR as a feedback of the channel to control the overall performance (IDR). A sample graph of IDR vs. CBR using modeling in [22] can be observed in Figure 2-3 which is in agreement with simulation observation in Figure 2-2. 


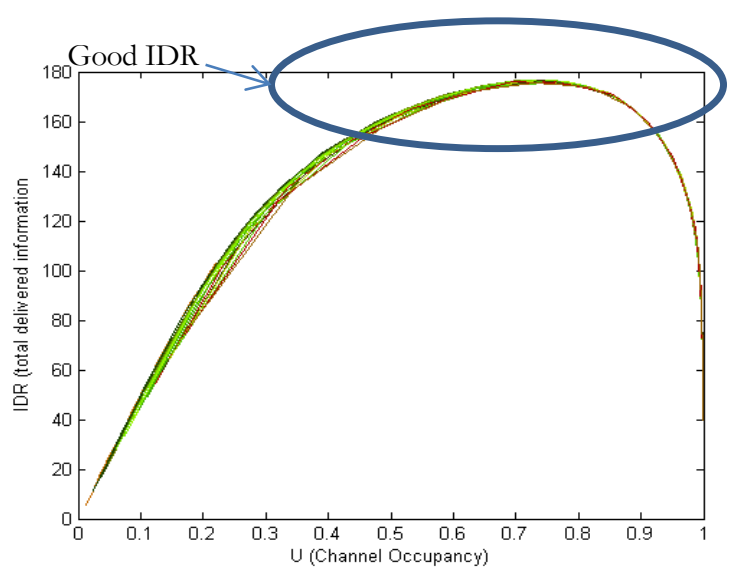

\section{Figure 2-3 Information Dissemination Rate for different rate (1-15) $\mathrm{msg} / \mathrm{sec}$ and ranges of transmission(20-400)m}

Next step would be to design an optimum controller for the system which maintains the IDR around its maximum. One suggestion for CBR support is [0.4 0.8]; this range can be tuned as long as it is not very relaxed to cause channel saturation.

Based on this observation a controller was designed for range control as depicted in Figure 2-4. This controller works based on the CBR calculated at each node as the feedback of the network and adjust the range of transmission respectively. The controller function can be observed in solid red line. This method is named Linear Memoryless Range Control (LMRC) and a more detailed description and analysis of the controller will be given in the following chapters. 


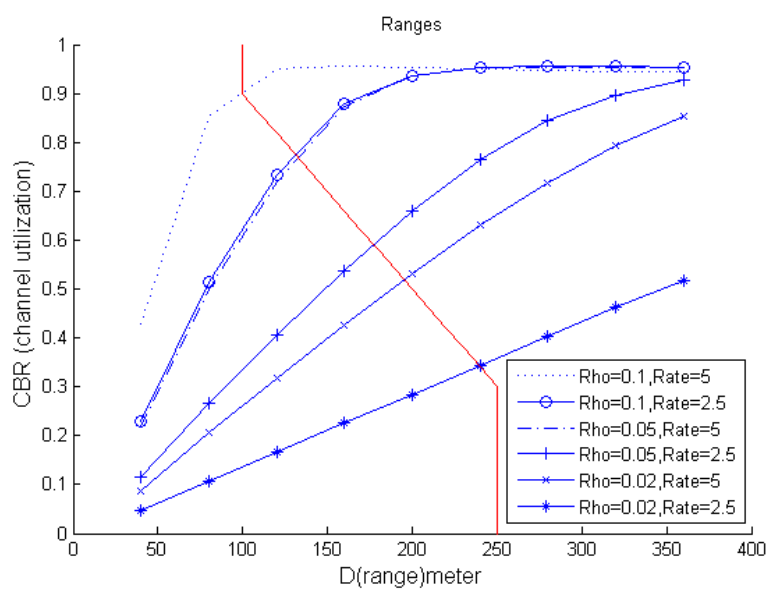

Figure 2-4 Range controller [21]

\subsection{Simulator Settings}

In this study we are using NS-3 network simulator which is licensed under GNU GPLv2 license and is mainly leveled for research and academic purposes. This open code network simulator is a discrete-event based simulator of the network which supports most of the extensions of IEEE 802.11(a/b/e/n/p) standard. IEEE 802.11p Medium Access Control (MAC) and Physical layer (PHY) to support different access categories have been used in this work. The main model of NS3 Wi-Fi can be observed in Figure 2-5 which is from nsnam website ${ }^{1}$. This figure contains the layers of MAC and Physical layer.

\footnotetext{
${ }^{1}$ http://www.nsnam.org/docs/release/3.10/doxygen/group__wifi.html
} 


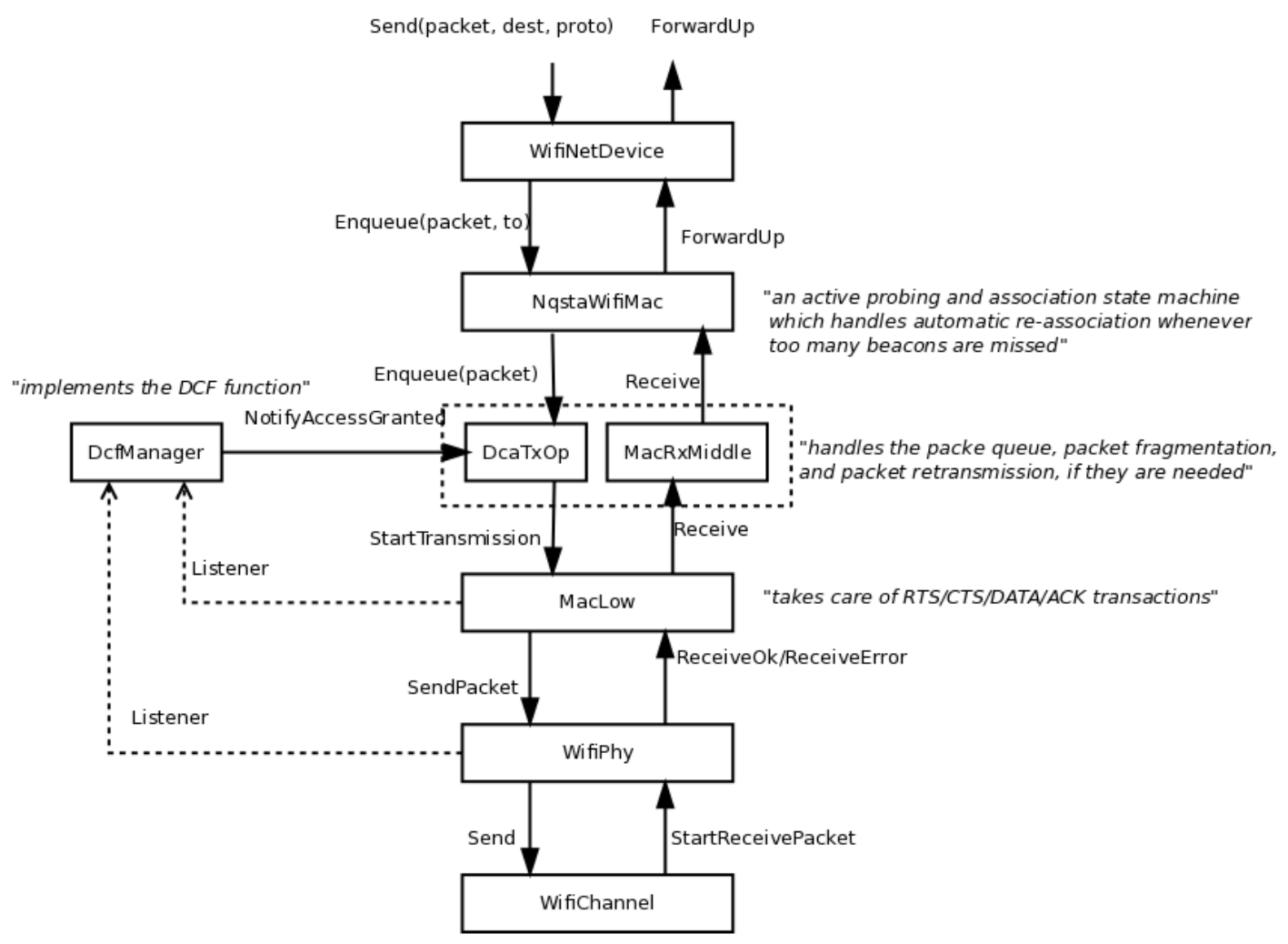

Figure 2-5 overview of Wi-Fi sub layers in NS3

We used an OFDM PHY for the $5 \mathrm{GHz}$ band with $10 \mathrm{MHz}$ channel bandwidth. The size of each packet was set to 500 bytes, sent at $5 \mathrm{~Hz}$ or $2.5 \mathrm{~Hz}$ based on the scenario being studied. Nodes use random traffic generation with the specified rate, which describes the case that adaptive control is being used and the sender will decide whether to send a packet or not based on vehicle movement. All the nodes will start randomly and since the broadcast is random too, it is the closest state to the reality. Study in [25] has been considered for the large scale channel modeling and fading models in the PHY layer which presents narrowband measurements of the mobile $\mathrm{V} 2 \mathrm{~V}$ propagation channel under realistic driving conditions. For the path loss a dual-slope model has been used corresponding the first data set studied in [25]. A Nakagami fading model is deployed which is more general than other models like Rician or Rayleigh fading models, and actually can represent wide range of 
fading situations, even probable conditions which are more severe than Rayleigh. Parameters used for the Nakagami fading model are an average based on data set 1 in [25] to consider the most realistic PHY layer in our simulations. In NS3 simulator there is possibility of choosing different fading models by variation of Nakagami parameters from Rayleigh $(m=1)$ to Rician $(m>1)$. A three step implementation in NS3 is implemented as depicted in Figure 2-6.

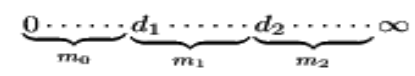

\section{Figure 2-6 distance bins for Nakagami fading model implemented in NS3}

The set of Nakagami parameters used for the simulation runs in this work, are as specified in Table 2-1. These values are chosen based on study in [25] which represents a Rician fading with $m_{0}=3.5$ for 5 meters around a vehicle and $m_{1}=2$ which is a Rician fading for distance from 5meters to 80meters, and for vicinities further than 80meters the fading model is more severe than Rayleigh with $m_{3}=0.7$.

Table 2-1 Nakagami fading parameters for simulation setup

\begin{tabular}{|c|c|}
\hline Parameter & Value \\
\hline $\mathrm{m} 0$ & 3.5 \\
\hline $\mathrm{m} 1$ & 2 \\
\hline $\mathrm{m} 2$ & 0.7 \\
\hline Distance1 & 5 \\
\hline Distance2 & 80 \\
\hline
\end{tabular}




\section{Chapter 3 : RANGE CONTROL ALGORITHMS}

As explained in previous section there are two controllable parameters in CVSS: 1) rate of transmission which specify frequency of message transmission 2) range/power of transmission that indicate the power of radio device to broadcast the message. In this letter we consider range/power control for CVSS and study existing algorithms presented in [13], in terms of temporal stability and convergence as well as spatial fairness . These algorithms are Linear Memoryless Range Control (LMRC) and Gradient Range Control (GRC) which is named based on its control function mechanism. In Linear Memoryless Range Control algorithm a range of good CBR which will result in acceptable IDR is considered Figure 2-2, this range is typically chosen around (0.4-0.8). This range for CBR values has been chosen based on operation of the channel in terms of throughput (IDR in this case) which is discussed in chapter 2.2. However this range of CBR will have a great effect on stability and fairness mechanism of the algorithm which was neglected in previous studies. A variation of LMRC algorithm is being implemented for industry and will be used in large scale networks of vehicles soon; therefore it is crucial to study the algorithm more carefully in terms of stability. The convergence conditions and stability of the LMRC algorithm is studied more sensibly here and we will suggest a few alterations to the algorithm to guarantee convergence for all typical road densities and rates of transmission. On the other hand, GRC algorithm has been thoroughly studied in [20] and will not be analyzed in this 
work but we will use GRC algorithm result for comparison purposes as well as fairness study.

\subsection{Linear Memoryless Range Control (LMRC)}

In CVS Systems (CVSS) achieving maximum awareness is critical which depends on the accuracy of cars estimation of their neighboring vehicles. This estimation is more robust when enough number of packets is received in a timely manner at the receiver. Therefore, it is important to maintain the throughput of the channel at an efficient level to avoid saturating the channel while using an effective capacity of the channel; this optimum range for throughput was previously argued to be achieved around CBR in interval (0.4-0.8) in chapter 2.2. Consequently Linear Memoryless Range Control algorithm obeys the aforementioned strategy to control the congestion by setting transmission power of nodes according to their sensed CBR. LMRC tries to maintain the CBR at a level that results in near optimal IDR. This is done by controlling the range/power of transmission according to CBR value. Figure 3-1 shows an example of this control function (solid line in red) as well as network characteristic curves for different typical rates of transmission and road densities. It should be noted that the road densities shown in Figure 3-1 are for each lane and the total density of cars on the road, which was an 8-lane highway (4-lanes in each direction), is eight times the shown value. 


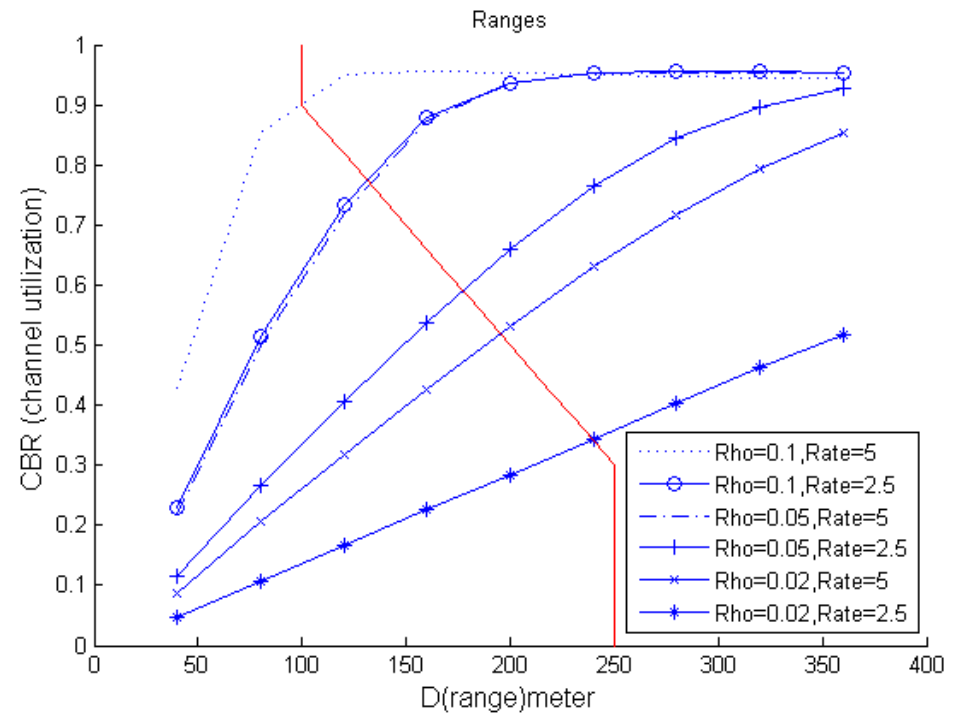

Figure 3-1 Communication characteristic curves for six different scenarios, and
feedback control function for range control [21]

The control function plotted in Figure 3-1 intersects all the curves in a range of CBR that yields good IDR and should bring the system to the intersection of the two graphs: the controller described by $D_{k+1}=f\left(U_{k}\right)$ and the network characterization graph presented as $U_{k}=g\left(D_{k}\right)$.There are many different choices for this controller depending on range of CBR and limits of transmission range. The general form of Linear Range Control (LRC) algorithm presented in [20] is as follows:

$$
D_{k+1}=f\left(U_{k}\right)=\left\{\begin{array}{lr}
D_{\max } & U_{k}<U_{\min } \\
D_{\min }+\left(\frac{U_{\max }-U_{k}}{U_{\max }-U_{\min }}\right) \times\left(D_{\max }-D_{\min }\right), & U_{\min } \leq U_{k} \leq U_{\max } \\
D_{\min } & U_{\max } \leq U_{k}
\end{array}\right.
$$

In (3) $U_{k}$ indicates the value of CBR used for adaptation algorithm and the limits for range $\left(D_{\min }, D_{\max }\right)$ are obtained from the safety requirements which is desirable to be in range of (50-100) for the minimum and (250-300) for the maximum range [24] . In order to define limits for $\left(U_{\min }, U_{\max }\right)$ for this controller, looking at Figure 2-2 we can see that the transmission range should be such that CBR value stays in range of (0.4-0.8) in order to keep the IDR near its peak value and maintain a good performance in terms of throughput. 
In following section stability conditions of this algorithm is discussed and a set of limits have been proposed to guarantee convergence of LMRC algorithm over time.

\subsubsection{Stability Analysis of LMRC Algorithm}

In this section, we study the convergence properties of LMRC and suggest one set of limits which satisfies convergence properties. Here we present lemma 1 which defines the condition for convergence:

Lemma 1: Assuming that network density and average transmission rate stay unchanged, any range control algorithm that uses a decreasing function of $\operatorname{CBR}\left(D_{k+1}=f\left(U_{k}\right)\right)$ is stable in time and converges to a single value for range if the following condition is satisfied:

$$
\left|g\left(D_{k+1}\right)-g\left(D_{k}\right)\right|<\left|f^{-1}\left(D_{k+1}\right)-f^{-1}\left(D_{k}\right)\right|
$$

The convergence property follows from the fact that for the algorithm to converge, two subsequent steps of the algorithm in (3) should lead to smaller difference in the subsequent observed range or CBR which can be easily visualized in Figure 3-3. Lemma one can be written in terms of CBR as follows:

$$
\left|U_{k+1}-U_{k}\right|<\left|U_{k}-U_{k-1}\right|
$$

Using equations $D_{k+1}=f\left(U_{k}\right)$ and $U_{k}=g\left(D_{k}\right)$ and substituting them in (5), (4) is derived. Inequality (4) describes the condition which should be satisfied for different types of controller function specified by: $D_{k+1}=f\left(U_{k}\right)$. For LMRC, (4) can be simply interpreted as $f^{-1}$ being steeper than $g\left(D_{k}\right)$ in the entire range of values for $D\left(D_{\min }\right.$ to $\left.D_{\max }\right)$. 


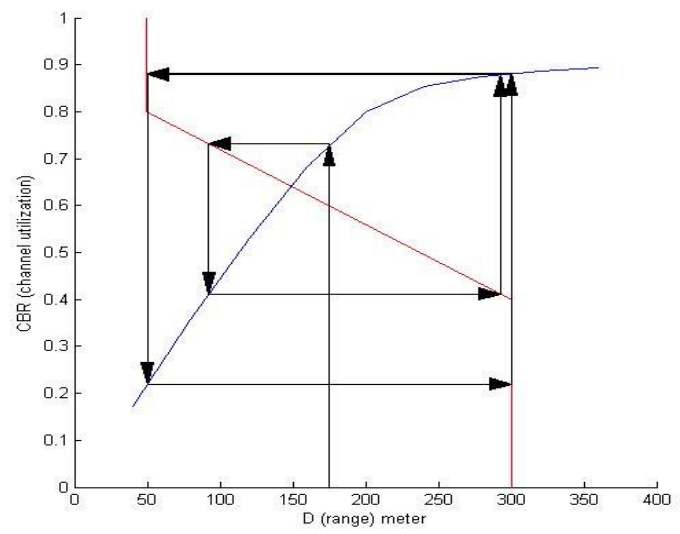

Figure 3-2 Divergence for LMRC algorithm for $\left(D_{\min }, D_{\max }\right)=(50,300)$, $\left(U_{\text {min }}, U_{\text {max }}\right)=(0.4,0.8)$ for $\rho=0.1$ and rate $=2.5$ scenario in MATLAB

The above condition is verified in a set of simulation runs in MATLAB, using Network Characteristic Curves (NCC) that was derived from NS-3 simulations. Based on our studies on convergence of LMRC we observed that desired limits cannot lead to convergence for all scenarios Figure 3-2. This is basically because in some scenarios the slope of the NCC is more than control function. Therefore, in order to satisfy the convergence condition we applied a slight alteration to the main control function as formulated in (6). This new formulation of the control function gives a more general control which could be tuned by setting parameter $\alpha$ to guarantee convergence for all possible cases. Subsequently by having the ideal range and CBR intervals [13][24] the general LMRC control function is introduced in (6) and it can be observed in Figure 3-3 in solid red line with a slight curvature for $\alpha=1.5$. This general version of the control function of the algorithm will allow a broader range for $\left(D_{\min }, D_{\max }\right)$ and $\left(U_{\min }, U_{\max }\right)$. The values used for these limits in this study for LMRC can be observed in (7). 


$$
D_{k+1}=f\left(U_{k}\right)=\left\{\begin{array}{lr}
D_{\max } & U_{k}<U_{\text {min }} \\
D_{\min }+\left(\frac{U_{\max }-U_{k}}{U_{\max }-U_{\min }}\right)^{\alpha} \times\left(D_{\max }-D_{\min }\right), & U_{\min } \leq U_{k} \leq U_{\max } \\
D_{\min }, & U_{\max } \leq U_{k}
\end{array}\right.
$$

Figure 3-3 shows the adaptation iterations for $\rho=0.1$ and rate $=2.5$ scenario, with controller parameters as in (7) and exponent $\alpha=1.5$ for the controller. This set of parameters for LMRC was found to result in convergence and stability in time for all the considered scenarios.

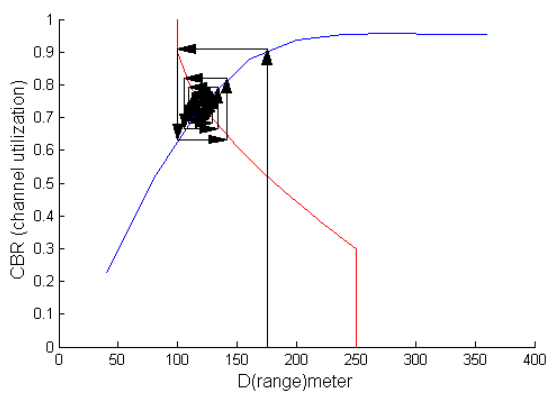

Figure 3-3 Convergence study on communication characteristic curve for $\rho=0.1$ and rate $=2.5$ scenario in MATLAB $[21]$

$$
\left\{\begin{array}{c}
\left(D_{\min }, D_{\max }\right)=(100,250) \\
\left(U_{\min }, U_{\max }\right)=(0.3,0.9) \\
\alpha=1.5
\end{array}\right.
$$

\subsubsection{Study of $L M R C$ for Static Highway Scenarios}

In this section the simulation result for the LMRC algorithm with the selected parameters of (7) are presented; this result shows the robustness of the algorithm for all the scenarios. Studied scenarios are presented in Table 3-1. In this table density of cars is defined as number of vehicles per one meter per lane; therefore, in case of n-lane highway the total 
density will be $\rho \times$ n. Subsequently a $\rho=0.1$ will present a very dense situation in which we have a car every $10 \mathrm{~m}$.

Table 3-1 studied scenarios

\begin{tabular}{|c|c|c|}
\hline Scenario & Density of cars $(\boldsymbol{\rho})$ & $\begin{array}{c}\text { Rate of } \\
\text { transmission }\end{array}$ \\
\hline 1 & 0.1 & 5 \\
\hline 2 & 0.1 & 2.5 \\
\hline 3 & 0.05 & 5 \\
\hline 4 & 0.05 & 2.5 \\
\hline 5 & 0.02 & 5 \\
\hline 6 & 0.02 & 2.5 \\
\hline
\end{tabular}

As it is seen in Figure 3-4, the algorithm converged quickly over time and remained stable for $\rho=0.05$ and rate $=5$ scenario. This is what we were expecting from the MATLAB simulations done in advance.
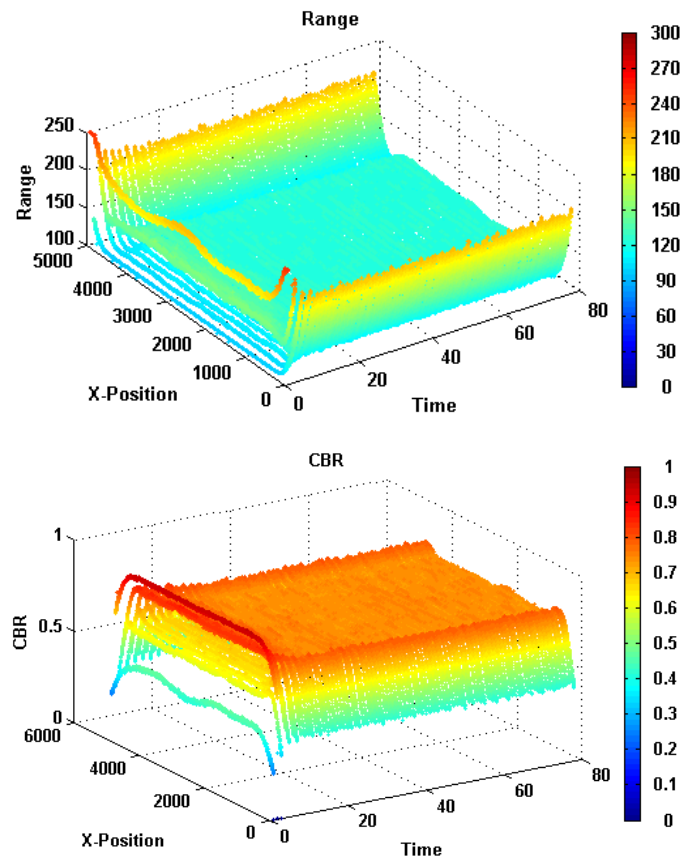

Figure 3-4 LMRC algorithm for $\rho=0.05$ and rate=5 scenario [21]

Moreover, rapid change in density is inevitable on highways which could happen in case of sudden jamming on the road because of an accident or road work. In either case the CVSS 
reliability should be assured to alert the drivers approaching the traffic jam about the situation. Therefore, a jam imitating scenario has been designed in which the density $\rho$ changes from 0.02 to 0.1 in the middle of the highway $(1500 \mathrm{~m})$. The algorithm manages to maintain time stability and space fairness as is seen in Figure 3-5. The LMRC algorithm shows solid performance in this case and can guarantee reliability in case of sudden jammed road.

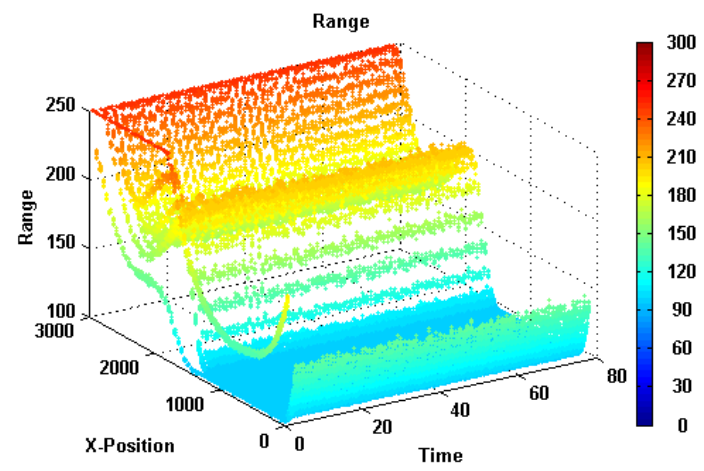

Figure 3-5 LMRC algorithm result for a mixed scenario which has a $\rho=0.1$ up to 1500 meter of road and $\rho=0.02$ for the next half. [21]

With the selected parameters, LMRC algorithm is able to maintain stability over time and fairness in space; therefore this algorithm can be a reliable candidate for congestion control. At time of this study a variation of LMRC algorithm is undergoing field test to pass the final verification steps for entering market. However, we should keep in mind that the selected controller should satisfy (4), which will limit our choices for $D_{\min }, D_{\max }$ and $U_{\min }, U_{\max }$. For example by reducing the slope of the control function and changing the limits to $(50,300)$ for range and $(0.4,0.8)$ for $\mathrm{CBR}$, we cannot achieve convergence anymore (see Figure 3-6 for example). If such limits are not acceptable in a specific design, an alternative is to use the Gradient descent Range Control (GRC) algorithm which does not put any limits on maximum or minimum range. It should be noted that a stateful version of LMRC will be introduced in chapter 5 of this letter which is stable under any 
choice of parameters and can be tune to guarantee convergence for all typical road densities.

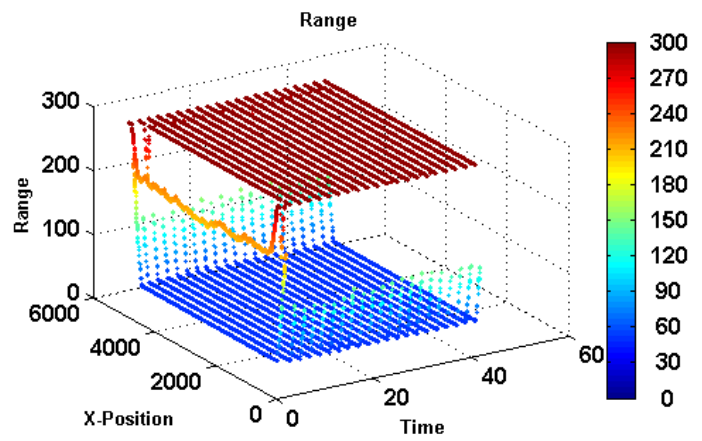

Figure 3-6 $\mathrm{LMRC}$ algorithm for $\boldsymbol{\rho}=\mathbf{0 . 0 5}$ and rate $=5$ scenario with limits $\left(D_{\min }, D_{\text {max }}\right)=(50,300)\left(U_{\min }, U_{\text {max }}\right)=(0.4,0.8)$, the algorithm does not converge in time result showing the range chosen by each node. [21]

In next chapter GRC algorithm will be introduced that uses a gradient descent method to adapt range of transmission by maintaining CBR value close to optimum CBR which is around 0.7 .

\subsection{Gradient Descent Range Control}

While LMRC tries to maintain CBR in a desired range, another alternative is to design an iterative gradient descent based algorithm that tries to maintain CBR at a specific optimal point (e.g, at 0.7). The update function of the algorithm can be introduced as follows;

$$
D_{k+1}=\min \left(D_{\max }, \max \left(D_{\min }, D_{k}+\eta\left(U^{*}-U_{k}\right)\right)\right)
$$

Where optimal CBR is denoted as $U^{*}=0.7$.The value of gain $(\eta)$ in this equation has been derived in [13] for the feedback linearized version of the algorithm that uses the following update function:

$$
D_{k+1}=D_{k}+\eta \times \ln \left(\frac{1-U_{k}}{1-U^{*}}\right)
$$

A short summary of the authors in [13] will be discussed in next chapter to analyze stability of GRC algorithm. 


\subsubsection{Stability Analysis of GRC algorithm}

Convergence properties of GRC were discussed in [13] and it was recommended that gain $(\eta)$ should be selected in a way that 2 conditions are met: 1) range $d$ converges quickly to near optimal value, before the value of $\rho$ or average value of rate changes considerably. 2) the system does not overshoot too much or oscillate and stray into a region that yields significantly low value of IDR (e.g. $C B R>0.95$ or $C B R<0.3$ ) [13]. In this algorithm the range maximum and minimum can be set more liberally which is chosen to be 100 meter for minimum and 300 meter for maximum range in this study.

The gain or $\eta$ value as stated by the authors in [13] can have different values in range of (10-200). The range of acceptable gains are analyzed in MATLAB simulation, using network characteristic curves that were obtained from NS-3, to find a good value of $\eta$ that stabilize all scenarios. It is observed that using a higher gain $\eta$ which is in acceptable range of (10-200), will result in faster convergence; however, we observed in NS-3 simulations that although the system got very quickly to stable point, on the contrary in some scenarios the system gets into an unfair situation in space. This happens because of the edge effect that propagates inside; this issue will be explained in more details in chapter 6 . On the contrary by choosing smaller values for $\eta$ convergence happens more slowly (still less than 10 steps[13]), but system acts more solidly and unfairness in space can be controlled by a distributed CBR measurement approach on top of congestion control.

After an extensive set of experiments, we found a gain value around 50 to be appropriate. It can be observed in Figure 3-7 that this value will support convergence to the optimal value $\left(u^{*}=0.7\right)$ in less than 10 steps. This interval is reasonable since changes in density of cars on the road and rate of transmission of vehicles seldom happen in extremely short time; if 
the algorithm runs at $1 \mathrm{~Hz}$ rate (which is a low rate) the convergence will happen in $10 \mathrm{sec}$ and road density changes are not usually excessive in 10sec interval. Therefore, in this study $\eta=50$ has been used for our GRC simulations in NS-3.

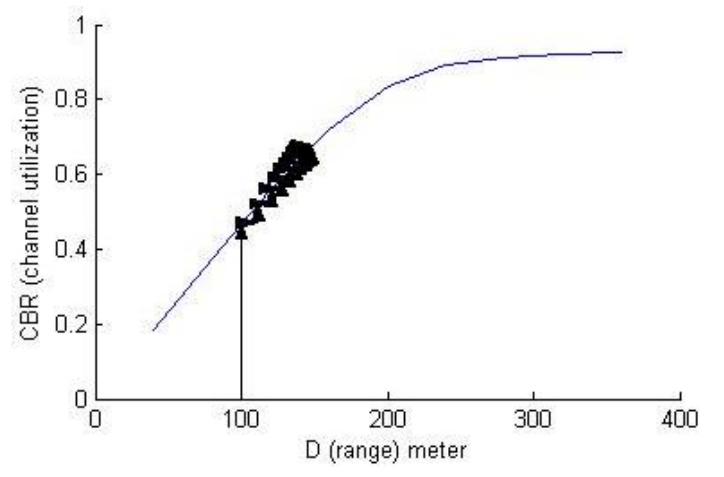

Figure 3-7 convergence steps for GRC algorithm for scenario with $\rho=0.05$ rate $=5$ and $\eta=50$ in MATLAB. [21]

For very dense and free flow scenarios $(\rho=0.1,0.02)$ GRC worked perfectly and stability in time and space was observed. However for $\rho=0.05$ and rate $=5$, we saw unfairness in space which is shown in Figure 3-8. We believe this is in fact the edge effect that propagates inward from the two edges. Edge nodes will have a lower local CBR sensed and set their range to maximum, causing higher CBR readings for the neighboring nodes. If this happens before other neighboring nodes are operating near their optimal point, they will sense the higher CBR and keep their range to lower values, even to $D_{\min }$ in some cases. This phenomenon will propagate inward throughout the network as seen in Figure 3-8, creating a ripple of low and high range (or high and low CBR), while road density is the same (all nodes should have had the same range). In order to solve this issue a solution is explained in chapter 6 of this work based on distributed measurement of CBR. 


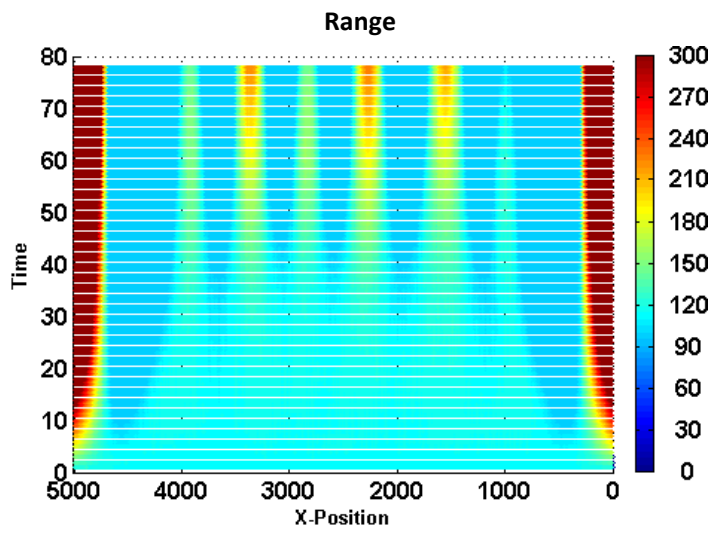

Figure 3-8 Result of GRC algorithm with $\eta=50$ for case $\rho=0.05$ and rate $=5$, unfairness is observed in this case [23]

It should be noted that in order to assure fairness in space, the distributed method for CBR measurement named averaging, is used on top of GRC algorithm for some of our simulation runs. The detailed explanation of averaging method is in chapter 6 of this letter. This distributed measurement is named averaging and is explained in more details in chapter 6.

\subsection{Study of Congestion Control Algorithms at Intersections}

In this section we have studied temporal stability and spatial fairness of the algorithms (LMRC, GRC) for intersection scenarios. Awareness at intersections is crucial since drivers have very limited line of sight and this shortage can be sizably compensated by CVSS to guarantee a safer drive. In order to study intersection scenarios, distinct characteristics of intersections should be taken into account to assure a robust study. The significant attributes of intersections to be noted are as follows: 1) being mostly congested at intersecting point and less at further spots 2) The packets will be highly affected by obstructing buildings or foliage at the blocks. In this study the first specification is considered by designing a scenario which resembles intersection setup, which means less dense at further places and more dense at intersecting area; the second characteristic, which 
is high shadowing effect by obstructing buildings at the blocks, is considered using parameters derived by the authors in [25]. Cheng et. al. in [25] study a typical urban scenario in Pittsburgh suburb which consists of few intersections as well. Therefore, we use derived parameters in that study for channel model, to calculate propagation loss. Accordingly the simulations are executed considering close conditions to real intersections propagation model which takes into account the high shadowing and high obstruction caused by these buildings and foliage on the road side.

Accordingly we have done a large number of simulations in NS-3 with Nakagami fading model and gradual increase in density up to intersection spot. A typical urban intersection such as a 4-way intersection is considered. In each leg of the intersection, 4 lanes exist with 2 lanes in each direction. First static trajectory files are exercised and in next chapter, verification of the result in realistic scenario will be presented. In the static trajectories used, there are two perpendicular streets (West-East and South-North), and their intersection is at point $(600,600) \mathrm{m}$. We have studied two kinds of static scenarios: 1) The most congested scenario with $\rho=0.2$ throughout the street 2) The other scenario is defined to imitate the intersection condition as close as possible. This more realistic scenario reflects a dense case at the intersection in each way $\rho=0.2$ for 100 meters $\rho=0.1$ for the next 100 meters and $\rho=0.05$ for the last 400 meters; the last part is selected to be 400 meters to avoid the edge effect as much as possible. Our main focus is to study the range control algorithms performance, so the rate of transmission is fixed and set to $2.5 \mathrm{and} 5 \mathrm{~Hz}$ based on previous studies in [20][21].

The simulation result for this case can be observed in Figure 3-9. In this figure a snapshot of the result is presented at time $=30 \mathrm{sec}$. The nodes at intersection point with higher density 
are choosing lower ranges of transmission while as it gets further from intersecting point the selected range increases, which is according to adaptation algorithm.
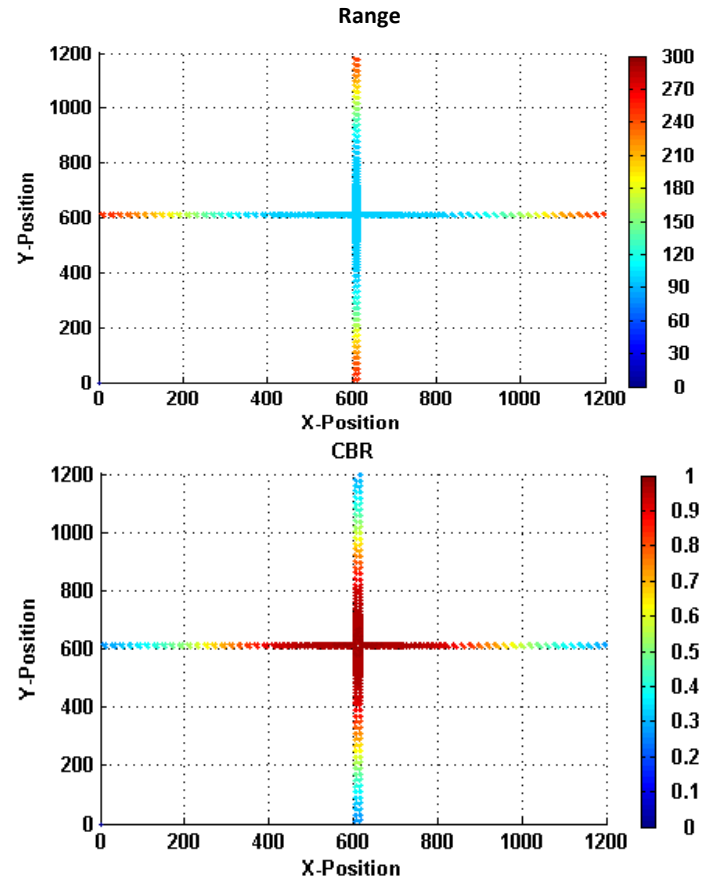

Figure 3-9 Snapshot at time $=30$ of $\mathrm{LMRC}$ Algorithm for mixed scenario with high density $(0.2-0.1-0.05)$ and rate $=5[23]$

In next section a complete set of results has been presented and analyzed.

\subsubsection{Result of Linear Memoryless Range Control Algorithm in Intersection Scenario}

By setting the LMRC parameters as indicated in (7) the algorithm worked robustly for all the scenarios. The results are shown for the West-East street in Figure 3-10 and afterward figures in this chapter; because of the symmetry of the road density in these scenarios, the South-North results are pretty much the same as West-East (see Figure 3-9 for example). So only the West-East street results are shown. Looking at Figure 3-10 we can observe that LMRC algorithm converges very fast for the most congested case. The fairness in space is satisfied too. 


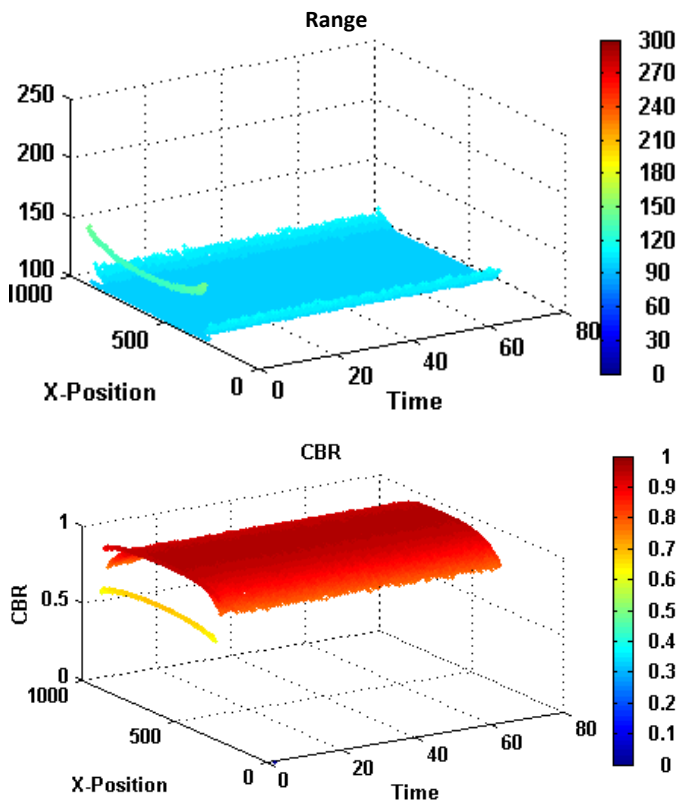

Figure 3-10 Result of LMRC algorithm with parameters in (5) for case $\rho=0.2$ and rate $=5[23]$

In order to consider a more realistic intersection scenario, we tried the mixed scenario as well. The results are shown in Figure 3-11. In this case the density is not the same for the entire road. However the algorithm has converged really quickly and the adapted range dedicated to the cars in each section of the road is according to its density and is stable in time and space. This shows the robustness of this algorithm to the density changes, which is a common phenomenon in real intersection scenarios. The range allocated to vehicles with higher density ( $\rho=0.2)$ near the intersection is the minimum (100 meter). Selected range for the further cars with lower density ( $\rho=0.1$ to $\rho=0.05$ ) has been set to higher values ending up to the maximum range (250 meter) for the nodes in the lowest density area. 


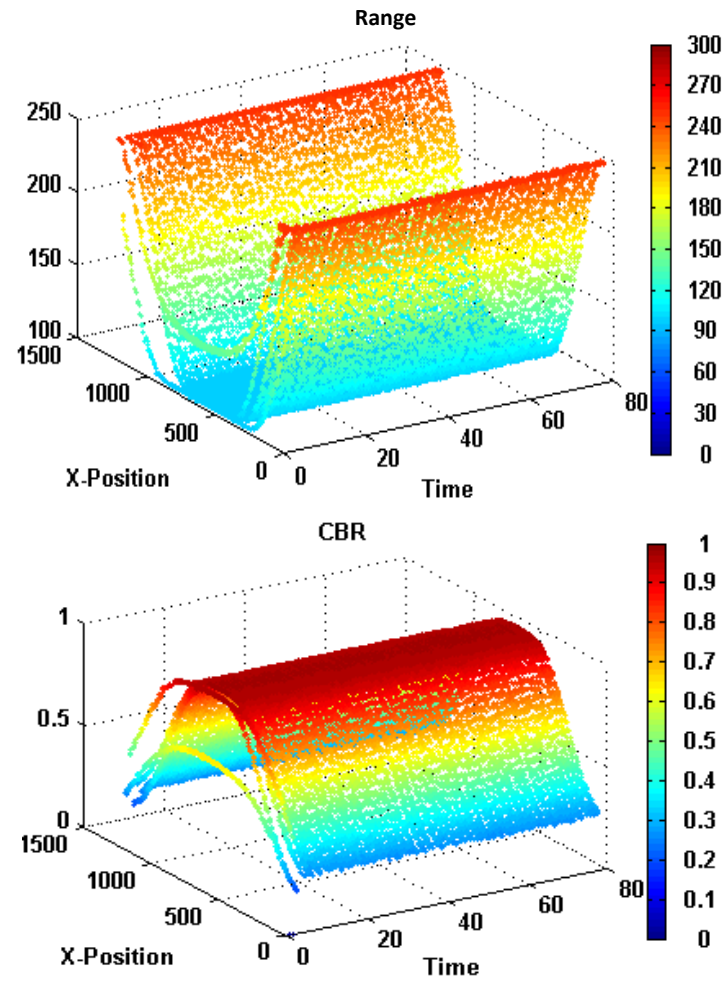

Figure 3-11 Result of LMRC algorithm with parameters in (7) for mixed scenario with high density $(0.2-0.1-0.05)$ and rate $=5[23]$

We should remember that the LMRC is working while satisfying (4), and this can limit our choices. By choosing different set of parameters instead of (7) this algorithm might lead to unstable conditions [21]. The Gradient descent Range Control (GRC) algorithm does not apply any constraints on minimum and maximum range and can be used as an alternative in cases that LMRC is not satisfying its convergence condition (4). Convergence requirements for GRC algorithm are presented in [13] and the maximum and minimum range was chosen 100-300 meter in this study. We will exercise this algorithm for the intersection case and show the results in the next section.

\subsubsection{Result of Gradient Descent Range Control Algorithm in Intersection Scenario}

As explained before the value for gain $(\eta)$ is chosen to be a low value near 50 . This selected gain will assure stability in time as well as fairness in space and try to prevent the 
edge effect from propagating inward; however we use averaging on top of GRC algorithm to assure fairness in space. Considering these facts we ran our experiments in NS-3 by setting the GRC algorithm gain to 50. In Figure 3-12 the result for the $\rho=0.2$ can be observed. The convergence happened very quickly and stability in time and fairness in space is achieved. Even in this case the edge effect can be observed at nodes further from the intersection point $(600,600)$. However by setting the gain to a low value we prevented this phenomenon to propagate inward.

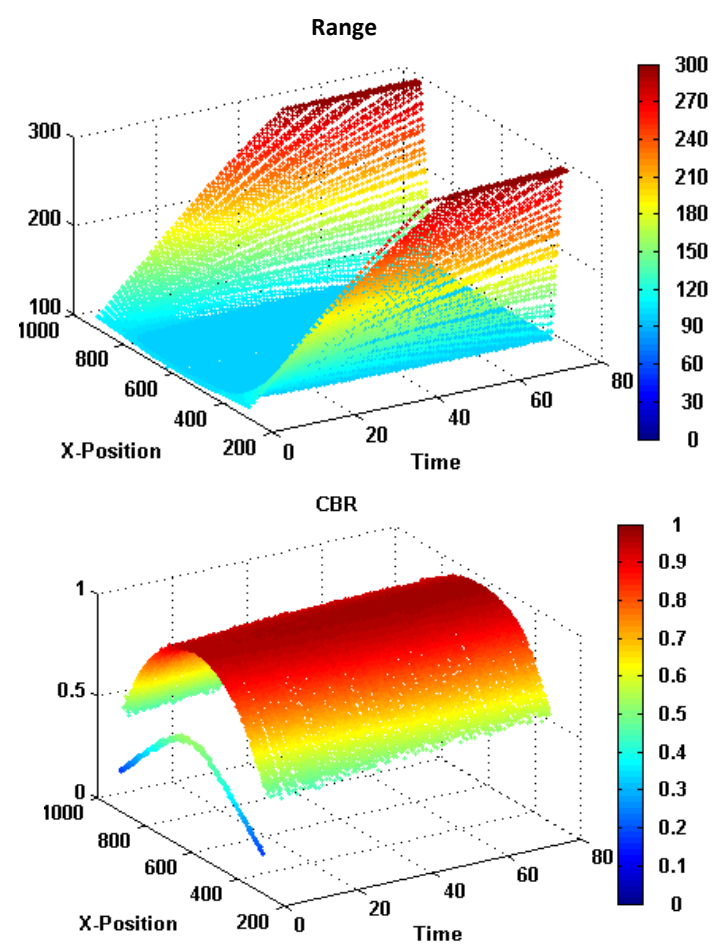

Figure 3-12 Result of GRC with $\boldsymbol{\eta}=\mathbf{5 0}$ for case $\rho=0.2$ and rate $=5$ [23]

The next scenario to study was the mixed density $(\rho=0.2)$ near the intersection and $\rho=$ 0.1 to $\rho=0.05$ for the farther points. The results are shown in Figure 3-13. As it can be observed, the stability in time and fairness in space is achieved.

In another scenario $\rho=0.1$ was used; the result is shown in Figure 3-14. For this scenario we had observed unfairness in a long highway scenario. However as it can be observed in 
Figure 3-12,Figure 3-13 and Figure 3-14 unfairness is not happening in this case. This is because of the shorter length of intersection scenarios comparing to highways.
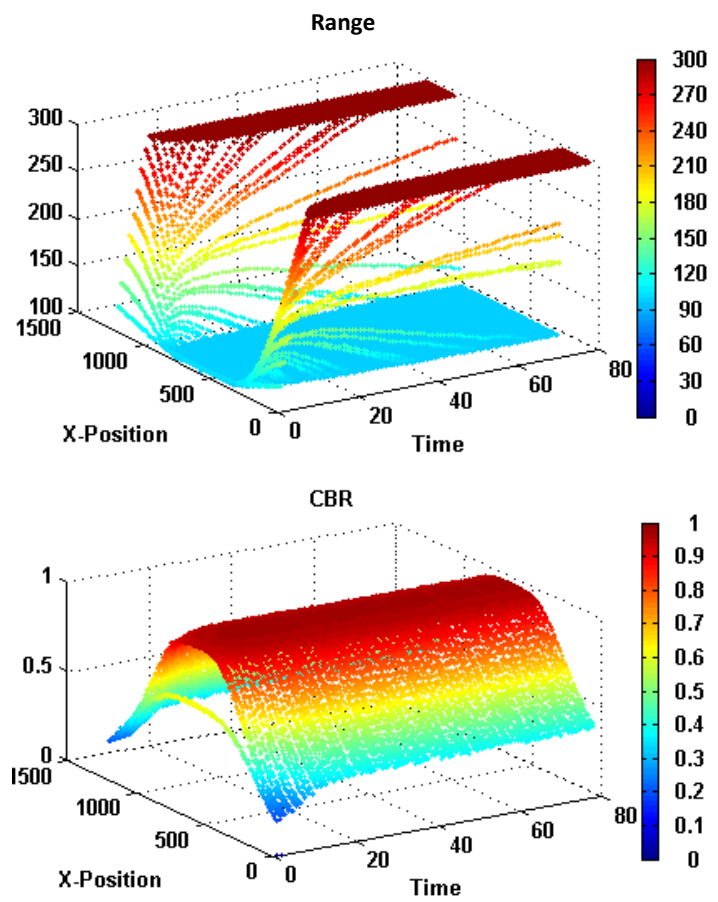

Figure 3-13 Result of GRC algorithm with $\eta=50$ for mixed scenario with high density $(0.2-0.1-0.05)$ and rate $=5[23]$
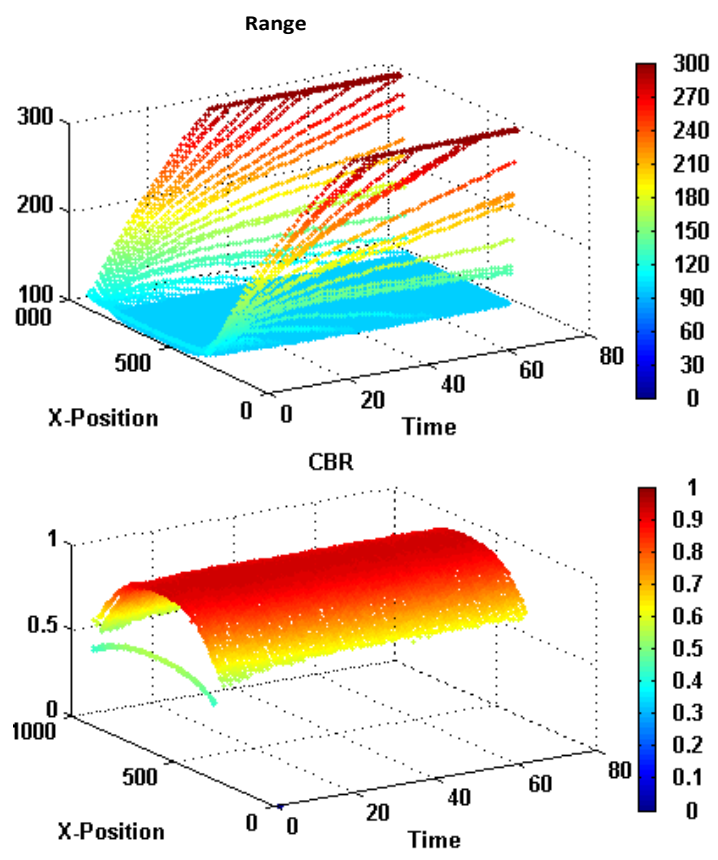

Figure 3-14 Result of GRC with $\eta=50$ for case $\rho=0.1$ and rate=5 [23] 
Nevertheless, unfairness is probable to happen as it was seen before, so in next set of results the distributed measurement of $\mathrm{CBR}$ is used. Using the distributed feedback measurement makes this study a solid method for different probable cases. Figure 3-15 and Figure 3-16 show the result for the same scenarios as Figure 3-12 and Figure 3-13. Looking at Figure 3-15 the effect of averaging can be observed for edge nodes; in the run of GRC with averaging the edge nodes are not setting their range to max range as it was observed in Figure 3-12.
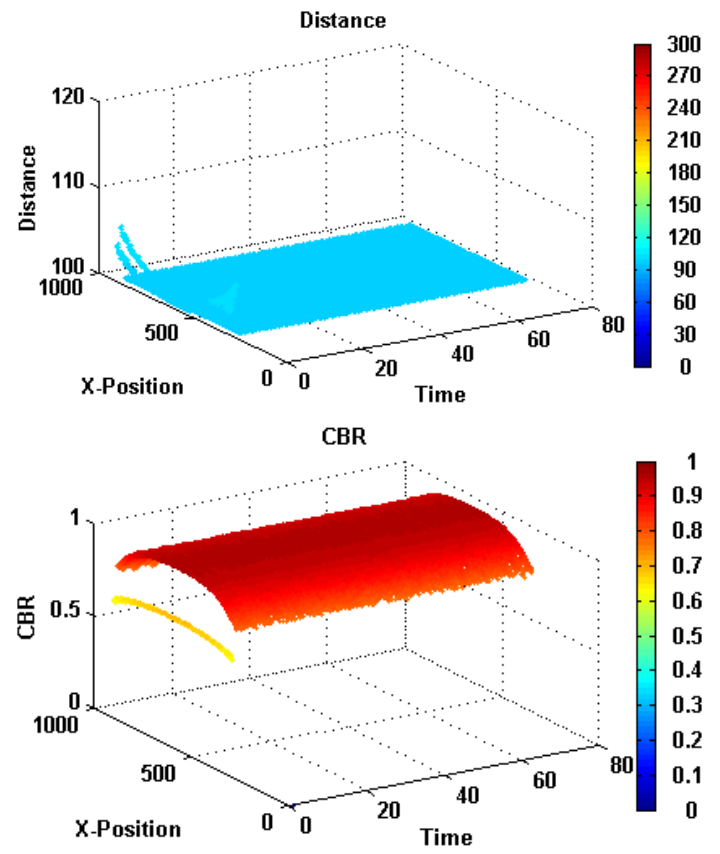

Figure 3-15 Result of GRC algorithm with averaging $\eta=50$ for case $\rho=0.2$ and rate $=5[23\rceil$ 

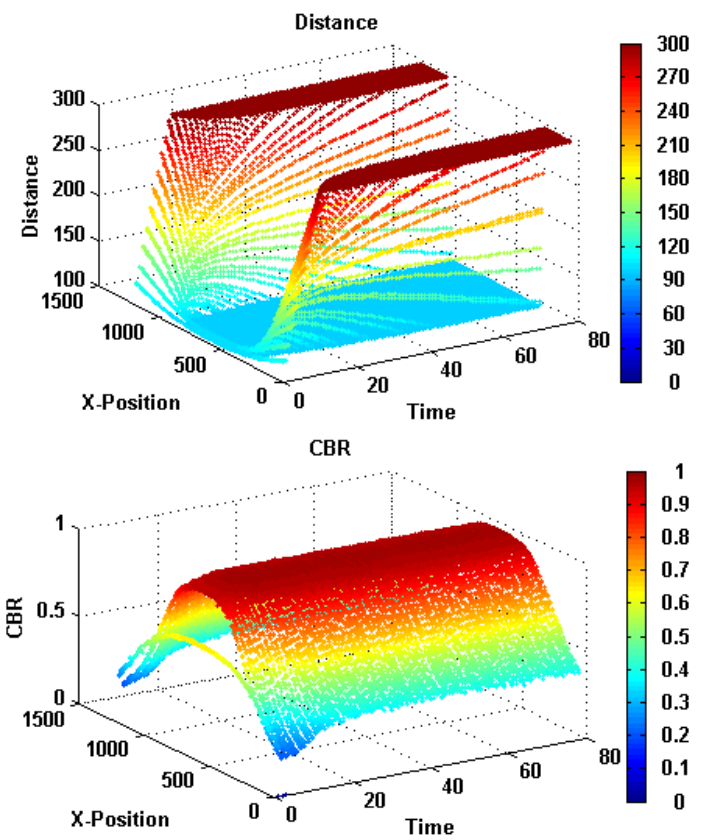

Figure 3-16. Result of GRC algorithm with averaging, $\eta=\mathbf{5 0}$ for mixed scenario with high density $(0.2-0.1-0.05)$ and rate $=5[23]$

\subsection{Verification in More Realistic Scenarios for LMRC and GRC}

\section{algorithms}

In this section more realistic trajectories are used which are derived from a traffic simulator named SUMO. This is done to show the robustness of the algorithm to movement of the nodes and assure the stability and fairness of the algorithm for moving vehicles.

\subsubsection{Highway Scenarios}

To verify the observed results from previous sections, we have considered more realistic situations. For this purpose we used SUMO trajectories for 3 different possible cases in a highway, from very congested to free flow. SUMO is an open source microscopic road traffic simulator. For these traffic simulations we have considered a 2000 meter 2-way highway which has 4 lines in each direction. The realistic scenarios used in this chapter are listed in Table 3-2 which covers most of probable scenarios. These cases are congested, 
low speed and free flow in which the maximum allowed speed for the vehicles are respectively 17,30 and $70 \mathrm{Mph}$.

Table 3-2 realistic scenarios description

\begin{tabular}{|c|c|}
\hline Scenario Name & Max speed \\
\hline Free flow & $70 \mathrm{mph}$ \\
\hline Slow Speed & $30 \mathrm{mph}$ \\
\hline Congested & $17 \mathrm{mph}$ \\
\hline
\end{tabular}

We set up some simulations to verify the LMRC algorithm for this set of typical realistic scenarios. The trajectories from the SUMO were fed into NS3 simulator using ns2mobilityHelper class and the results can be observed below. As you can observe the result for LMRC for the realistic scenarios are stable over time and fair in space.
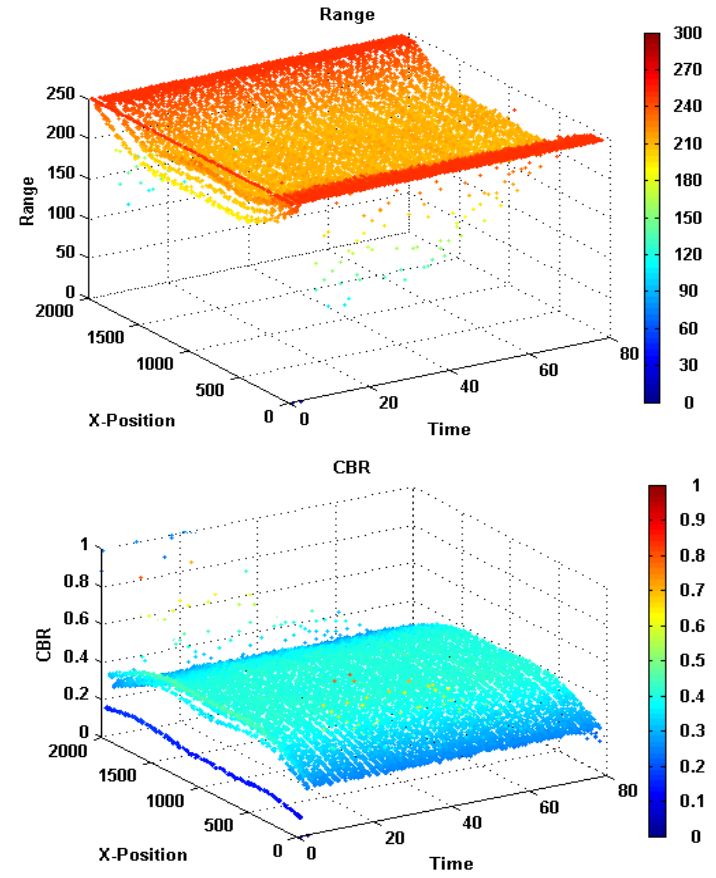

Figure 3-17 LMRC algorithm for free flow scenario (max speed= $70 \mathrm{Mph}$ ) [21] 
Looking at Figure 3-17 the stability in time is satisfied, however a slight effect of edge nodes can be observed in this scenario. The edge effect in LMRC algorithm is less severe than GRC algorithm because of the control function mechanisms.
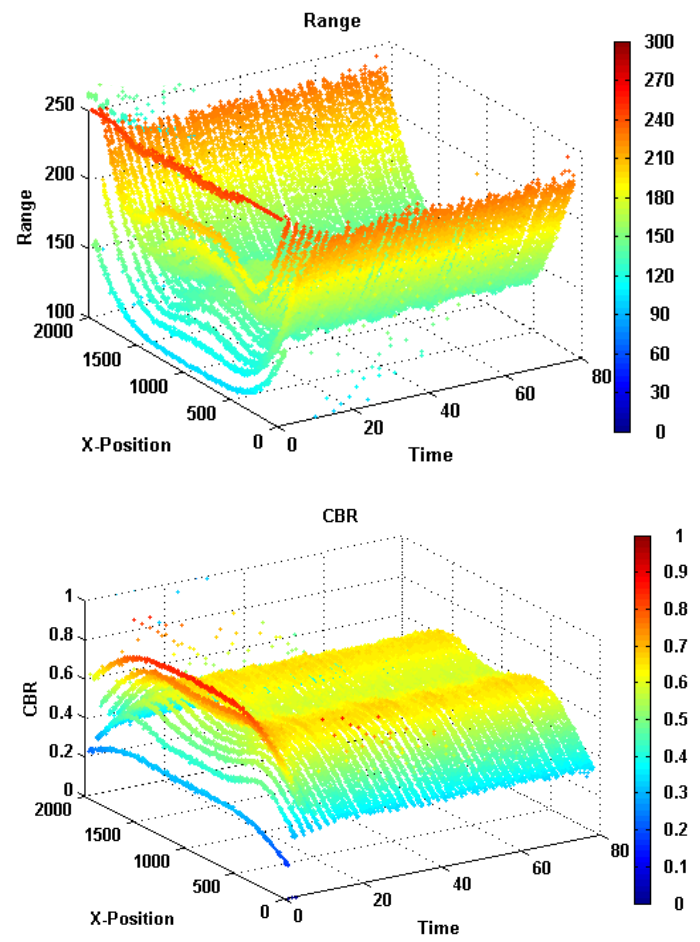

Figure 3-18 LMRC algorithm for low speed scenario (max speed= $30 \mathrm{Mph}$ ) [21]

In Figure 3-18 the result for LMRC algorithm can be observed. The algorithm is stable in time and roughly fair in space. The little ripple in range values is not severe and is less than $10 \%$ of adapted range. This weakness, however not very serious, is resolved in new algorithm proposed in chapter 4 with using fairness mechanism discussed in chapter 5 .

\subsubsection{Intersection Scenarios}

For intersection we are using the same software (SUMO) to create more realistic trajectories for vehicles. The mobility files obtained from this simulator are fed to NS-3 
simulator for studying the results. The network topology considered for this work is a 500 meter $\times 500$ meter grid, intersecting at $(250,250)$, it has 6 lanes, 3-lanes in each direction. Two cases are studied 1) low density 2) high density; in low density scenario vehicles have the maximum speed of 70mph and less number of vehicles entering the intersection and in high density scenario vehicles maximum speed is $17 \mathrm{mph}$ and there are more number of vehicles set to enter the intersection to imitate a more dense situation. The speed is adjusted by SUMO according to traffic patterns. One sample scenario run in SUMO GUI can be observed in Figure 3-19.

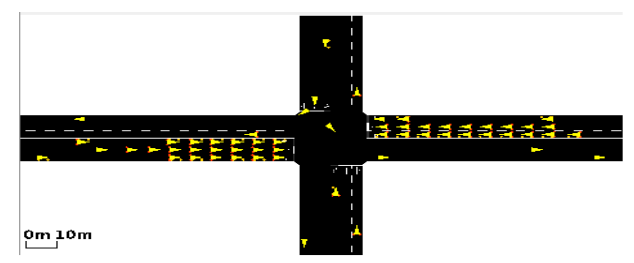

Figure 3-19 SUMO simulator GUI [23]

For these scenarios a higher gain $(\eta=100)$ has been chosen for GRC algorithm so the convergence speed can keep up with vehicles movement. The result for low density scenario is shown in Figure 3-20 and Figure 3-21 and the stability and fairness of the algorithm can be observed. The transmission range which is set for all the vehicles is the maximum which is 300 in this case with a quick convergence in time and stability in space is achieved as well. It should be noted that the edge effect is alleviated in this result by using averaging method. To explain the difference pattern of movement in Figure 3-21 for North-South street it should be mentioned that the flow of traffic from N-S street is lower than W-E street and after second 40 all the traffic from $\mathrm{N}-\mathrm{S}$ street are merged with W-E street. 

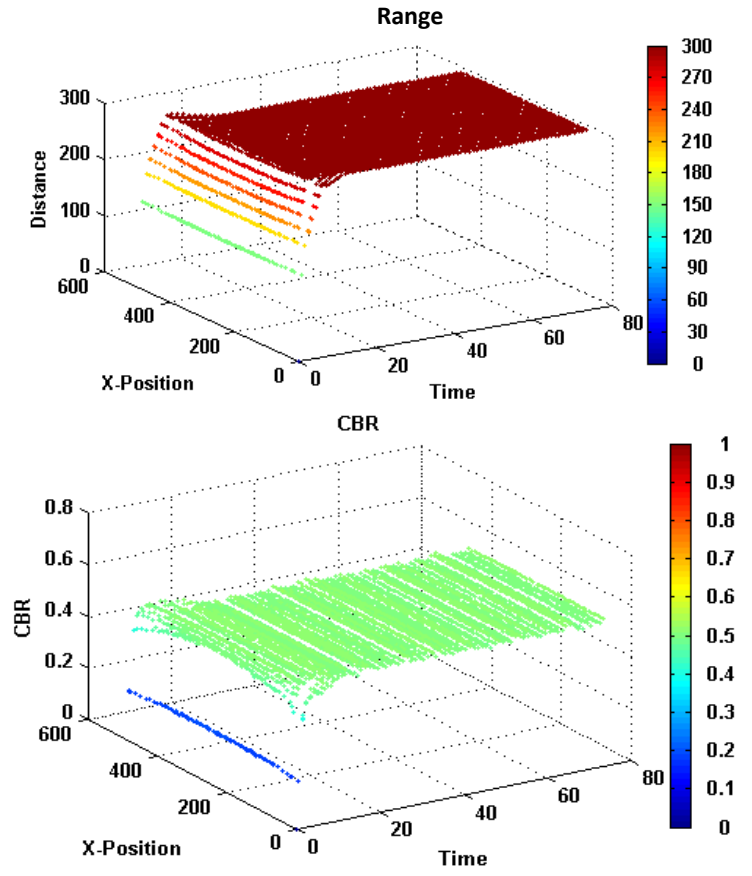

Figure 3-20 Result of GRC algorithm with averaging $\eta=100$ for SUMO low density in 2 ways scenario, East-West street, rate=5 [23]
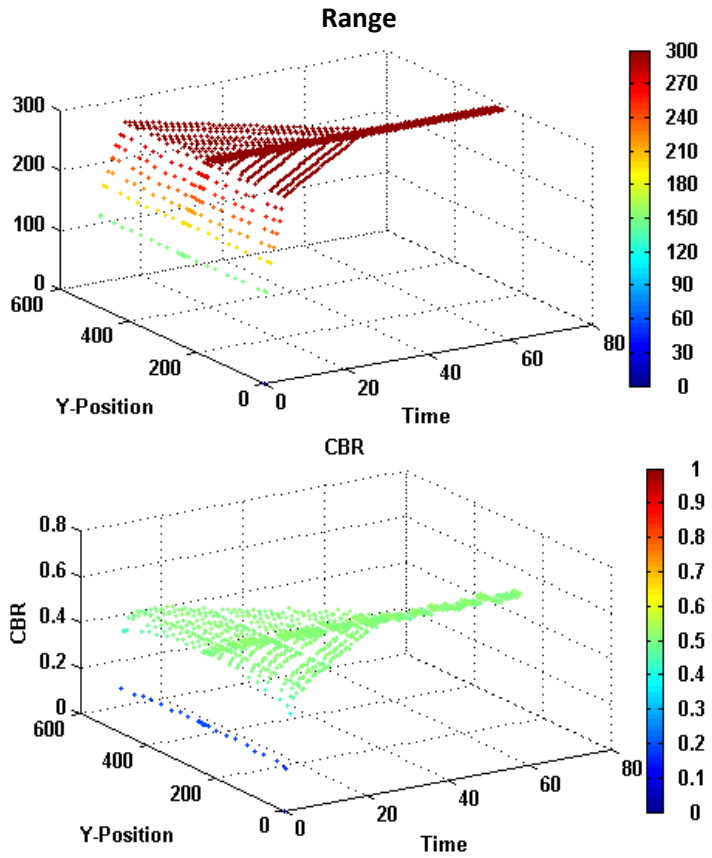

Figure 3-21 Result of GRC algorithm with averaging $\eta=100$ for SUMO low density in 2 ways scenario, South-North street, rate=5 [23]

The other studied scenario represents a high density road condition in all 4 ways and the maximum speed of $17 \mathrm{mph}$ is chosen. The stability of algorithm in time and fairness in 
space are achieved using the GRC algorithm. The results can be observed in Figure 3-22 and Figure. 3-23. As it can be seen as vehicles are moving toward the intersection (which has the higher density and consequently higher CBR is sensed) the range has been adapted quickly to the minimum distance. The results for both South-North and East-West look similar since the density is high in both South-North and East-West streets.
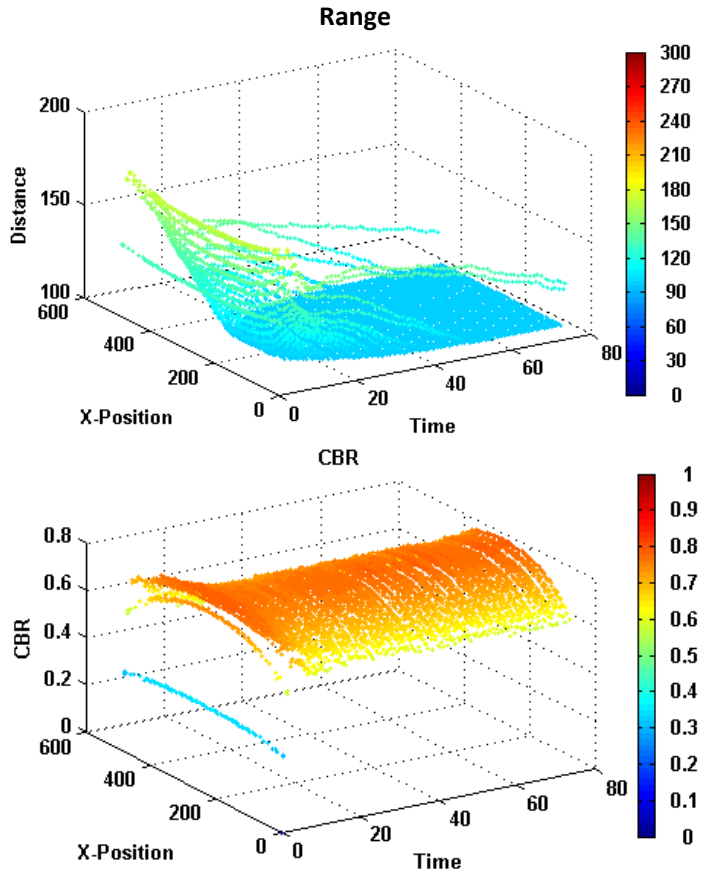

Figure 3-22 Result of GRC algorithm with averaging $\eta=100$ for SUMO high density in 4 ways scenario, East-West street, rate $=5$ [23] 

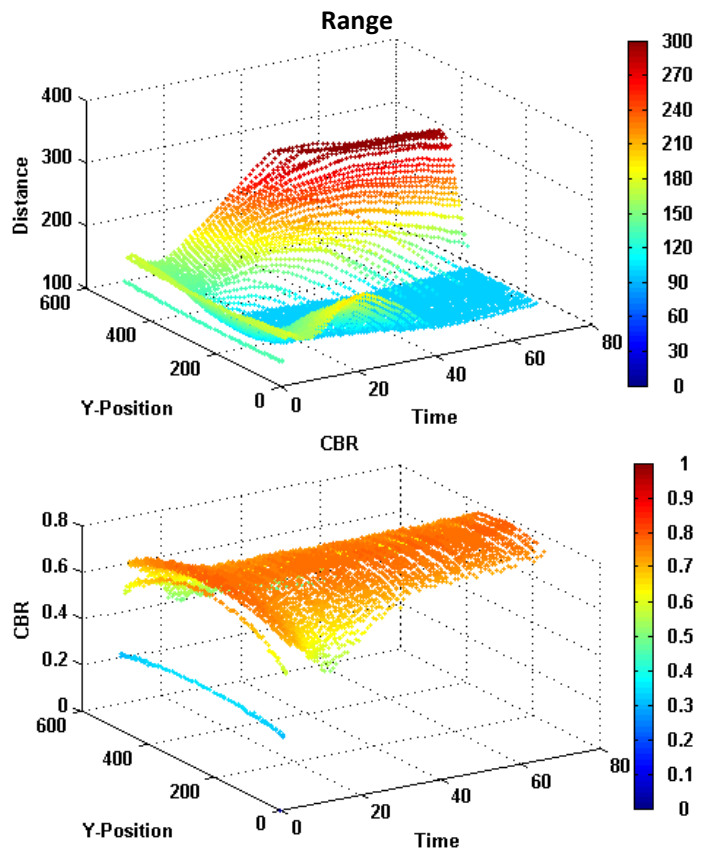

Figure. 3-23 Result of GRC algorithm with averaging $\eta=100$ for SUMO high densitv in 4 wavs scenario. South-North street. rate=5 [23]

The GRC algorithm results were promising and robust. Similar observations were made for LMRC (Figure. 3-24) with no CBR enhancement, as there was no need for distributed feedback measurement to achieve fairness. Stability for LMRC algorithm is insured by using parameters in (7). The result presented in Figure 3-22and Figure. 3-24 are both for high density case for East-West street using GRC and LMRC adaptation mechanism respectively and could be compared against each other. Both algorithms are stable and similar behavior can be observed for both GRC with averaging and LMRC since the selected range by both algorithms is minimum range. 

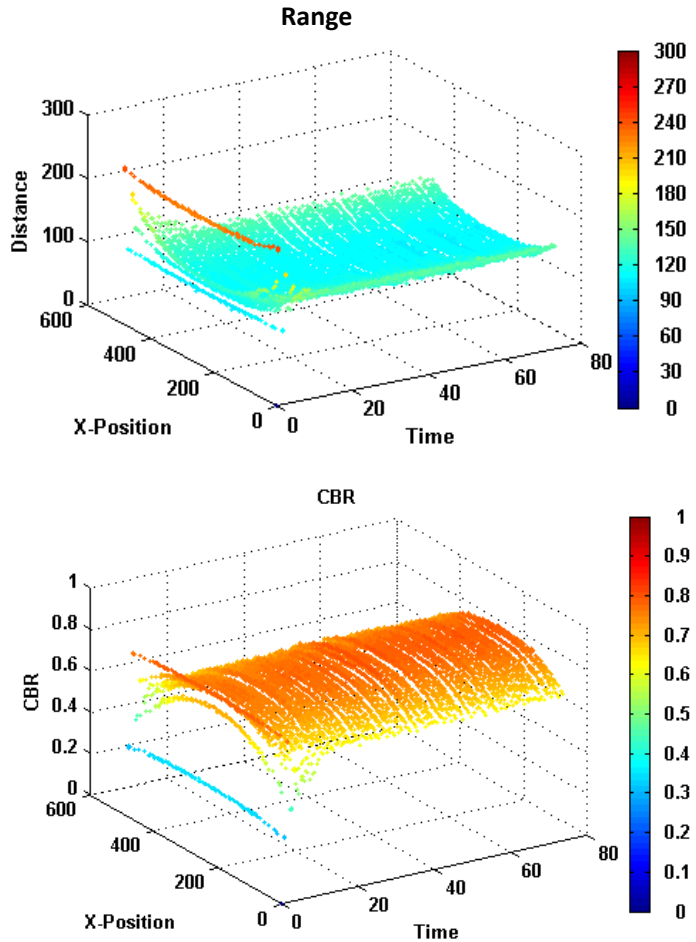

Figure. 3-24 Result of LMRC for SUMO high density in 4 ways scenario East-West street, rate $=5[23]$

In next chapter the novel power control mechanism is introduced which is a variation of LMRC algorithm without limitations. This new approach for power adaptation is an adaptive version of LMRC which adapts its operation using a gain. More detailed description and analysis of the algorithm will be presented in next chapter. 


\section{Chapter 4 : STABILIZED POWER CONTROL}

Based on previous studies on LMRC algorithm it was observed that this algorithm is dependent on limits defined for its control function (7). This fact could limit the option for minimum and maximum of transmission power and in worst scenario there might be probable instability. This was the motivation to come up with a stabilized power control design for the power adaptation to overcome these shortcomings of LMRC. Stateful Utilization-based PoweR Adaptation algorithm (SUPRA) was a result of this investigation. SUPRA algorithm uses the LMRC concept of linearly mapping CBR to Power, but controls the amount of change in each iteration using a configurable gain and a one-step memory. The gain can be adjusted such that the algorithm always converges to a solution in the desirable range of CBR values. We also opt for direct power control, rather than range control followed by mapping of range to power. This is because of two main reasons: 1) the limit of power for each device is indicated and by setting the power rather than range and then mapping it to corresponding power, we are sure to be in range of device limits during adaptation process 2) mapping of power to range is very much dependent on environment the device is operating; therefore, adjusting power directly could be more robust when all vehicles are doing the same power adaptation. In contrast if vehicles are doing range control followed by power mapping, they might have inconsistency in value of power since there is no unique power mapping for all the environments. Based on this logic, the direct power control is used for SUPRA and it will be introduced and analyzed in 
the following writing. The SUPRA algorithm works based on CBR feedback of the channel similar to LMRC and GRC.The algorithm can be explained using the following formula that sets the power following a measurement of the CBR $\left(U_{k}\right)$. Note that the formula uses the last set value of power $P_{k}$, to calculate $P_{k+1}$, thus becoming stateful.

$$
P_{k+1}=P_{k}+\eta \times\left(f\left(U_{k}\right)-P_{k}\right)
$$

where $f\left(U_{k}\right)$ is calculated as:

$f\left(U_{k}\right)= \begin{cases}P_{\max } & U_{k}<U_{\min } \\ P_{\min }+\left(\frac{U_{\max }-U_{k}}{U_{\max }-U_{\min }}\right) \times\left(P_{\max }-P_{\min }\right) & U_{\min } \leq U_{k}<U_{\max } \\ P_{\min } & U_{\max } \leq U_{k}\end{cases}$

This desired range of $\mathrm{CBR}$ is $(0.4,0.8)$, but power minimum and maximum $\left(P_{\min }, P_{\max }\right)$ values may now be arbitrarily set; the gain will then be adjusted to ensure the algorithm stays stable. The schematic of the algorithm can be observed in Figure 4-1.

The improvement over LMRC is seen through regulation of the difference between power settings in two consecutive steps. In LMRC, the new value for range (power) is selected as $f\left(U_{k}\right)$ which is the control function value at the measured CBR $\left(U_{k}\right)$. However in SUPRA the next value of power is calculated by finding the difference between $f\left(U_{k}\right)$ and $P_{k}$ (the last value of power) and then scaling the difference by gain $(\eta)$ and adding it to $P_{k}$. An appropriate value for the gain in the update equation ensures that the difference of two steps remains small enough so the algorithm converges to a solution. The next section studies how the gain affects the stability of the algorithm. 


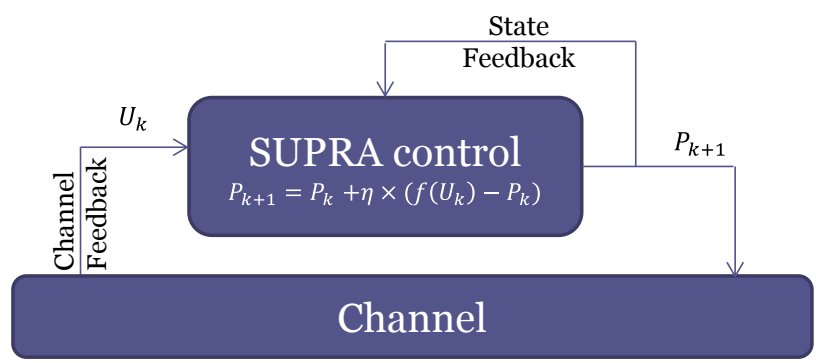

Figure 4-1 SUPRA control schema

\subsection{Stability Analysis of SUPRA}

To derive the stability conditions of SUPRA, and therefore finding the appropriate gain for the update equation, we study the dynamics of the algorithm in this section. The stability requirement should be studied for all possible and likely operation conditions. These conditions will characterize the set of network characteristic curves for which we study the SUPRA algorithm. Figure 4-2 shows a set of CBR vs. power curves (Network Characteristic Curve) that characterize the network (plant) behavior for a range of typical network settings in terms of the average transmission rate and density of nodes. Since increase in rate or density has almost the same effect, some extreme cases are also covered (e.g. $\rho=0.1$, rate $=10$ ). 


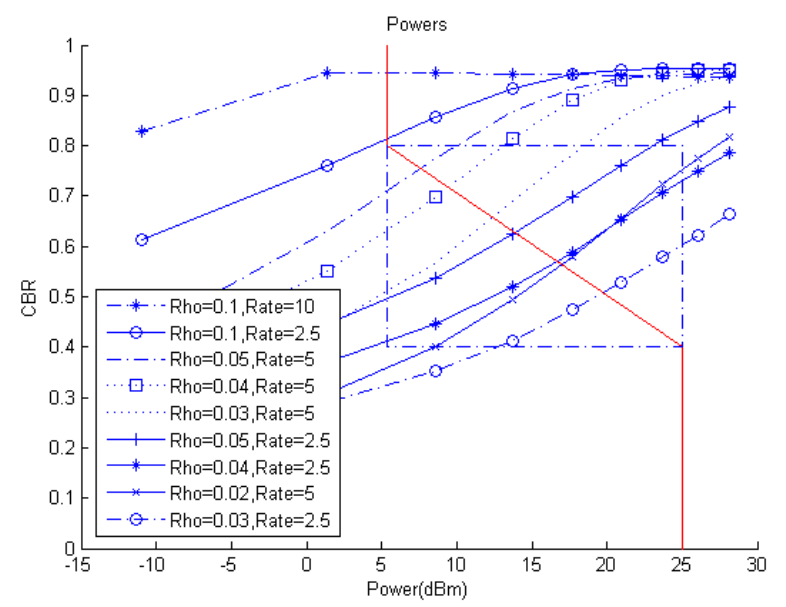

Figure 4-2 Communication characteristic curves for eight different scenarios, Power Control function (solid red line), Operational Area of the SUPRA-Control (dashed lined rectangle in blue)

The SUPRA algorithm is stateful and uses the previous value of power $\left(P_{k}\right)$ to find the appropriate new value. The operational area of the algorithm is shown in Figure 4-2 and is marked with dashed line. It can be observed from the same figure, that the network characteristic curves, for most typical scenarios in the operational area can be very well approximated by a linear function of sensed CBR, $g\left(U_{k}\right)$. The curves are similar for different typical scenarios with only slight differences in their slope. To study the stability of the algorithm, we use these reasonable linear approximated network characteristic curves in this section.

To study SUPRA stability we consider two consecutive steps of the Algorithm operation as plotted in Figure 4-3. The symbols showed in this figure are defined as follows:

$$
\begin{aligned}
& l_{1}=\left|P_{k}-f\left(u_{k}\right)\right| \\
& l_{2}=\left|f\left(u_{k+1}\right)-P_{k+1}\right|
\end{aligned}
$$

$f\left(U_{k}\right)$ : Main control function 
$f_{s}\left(U_{k}\right)$ : SUPRA control Function

$g\left(U_{k}\right)$ : Network characteristics curve approximation in operational area

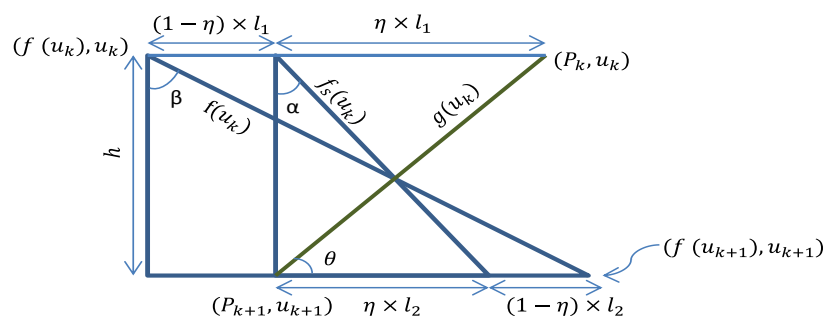

Figure 4-3 schematic illustration of SUPRA control on a sample network characteristic curve approximated as a linear function

The algorithm can approach the convergence point from both sides as shown in Figure 4-4(a) or it can be from one side, Figure 4-4(b). Case (b) will happen for $0<\eta<$ $\eta^{*}$, in which $\eta^{*}$ is the gain that makes the algorithm converges very fast and in one iteration so the value of $\alpha=0$. As the selected value for $\eta$ gets closer to zero the convergence will be slower.It is obvious that the algorithm will converge in this scenario, since the convergence condition (3) always hold. However for case (a) we need to find a good range for gain $(\eta)$ to guarantee convergence.
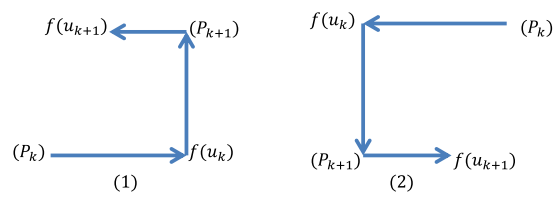

(a)
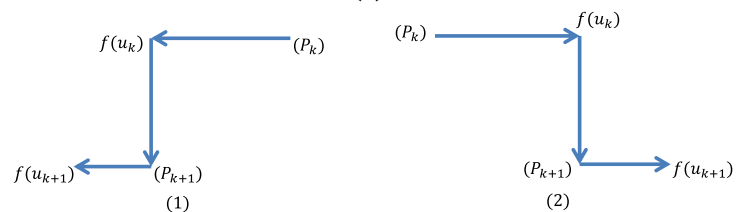

(b)

Figure 4-4 illustration of different states for two consecutive steps of control function 
It must be noted that the control functions are plotted in the figure showing network characteristic curves, so we see the inverse of $f\left(U_{k}\right)$ in Figure 4-2 and Figure 4-3 and inverse of $f_{s}\left(U_{k}\right)$ in Figure 4-3.

To illustrate the algorithm operation, we note that the gain $(\eta)$ used in SUPRA update function actually tilts the main control function $f\left(U_{k}\right)$ to what is shown as $f_{s}\left(U_{k}\right)$ in Figure 4-3. Looking at it this way, we can assume that we have the same control function as LMRC but with an adjustable slope which depends on the value of gain $\eta$. With this illustration we can apply the same convergence conditions derived for LMRC by some variation for this algorithm. The slopes of stabilized and main control functions are as below:

$$
\operatorname{tg}(\alpha)=\frac{\eta l_{2}}{h}, \operatorname{tg}(\beta)=\frac{(1-\eta) l_{1}+l_{2}}{h}
$$

So we can correlate the slope of $f\left(U_{k}\right)$ presented as $\operatorname{tg}(\alpha)$ and slope of $f_{s}\left(U_{k}\right)$ shown by $\operatorname{tg}(\beta)$ using following equation:

$$
\operatorname{tg}(\alpha)=\operatorname{tg}(\beta)-\frac{(1-\eta)\left(l_{1}+l_{2}\right)}{h}
$$

The second term can be rewritten, by substituting $l_{1}, l_{2}$ values, as follow:

$$
\frac{(1-\eta)\left(l_{1}+l_{2}\right)}{h}=(1-\eta)\left[\frac{\left|P_{k}-f\left(u_{k}\right)\right|+\left|f\left(u_{k+1}\right)-P_{k+1}\right|}{h}\right]
$$

We know that $l_{1}, l_{2}$ values are length so we have the absolute values in (12). In order to eliminate absolute values we should look at different states of two consecutive steps of the control. This is illustrated in Figure 4-4(a). 
Two possible cases for each two consecutive steps of the algorithm can be considered as follows:

$$
\left\{\begin{aligned}
f\left(u_{k+1}\right)>P_{k+1}, & f\left(u_{k}\right)<P_{k} \\
f\left(u_{k+1}\right)<P_{k+1}, & f\left(u_{k}\right)>P_{k}
\end{aligned}\right.
$$

Theoretically we can then rearrange the absolute values in (12) and by a little bit of tweak, following equation will be attained:

$$
\frac{(1-\eta)\left(l_{1}+l_{2}\right)}{h}=(1-\eta)\left[\left|m_{g^{-1}}\right|+\left|m_{f}\right|\right]
$$

In which $m$ is the slope of the function. Finally we can rewrite (11) as:

$$
\operatorname{tg}(\alpha)=\operatorname{tg}(\beta)-(1-\eta)\left[\left|m_{g^{-1}}\right|+\left|m_{f}\right|\right]
$$

On the other hand the convergence condition in (4) can be rewritten in terms of Power as follows:

$$
\left|P_{k+2}-P_{k+1}\right|<\left|P_{k+1}-P_{k}\right|
$$

This equation (16) can be rewritten in terms of stabilized control function values, considering Figure 4-3 as below:

$$
\left|f_{s}\left(u_{k+1}\right)-P_{k+1}\right|<\left|f_{s}\left(u_{k}\right)-P_{k}\right|
$$

Having the definition of tangent value in a right triangle which is the length of opposite by adjacent edge and looking at Figure 4-3 we can write (17) as:

$$
\operatorname{tg}(\alpha)<\operatorname{tg}\left(\frac{\pi}{2}-\theta\right)
$$

It can be observed from the same figure that: 


$$
\operatorname{tg}\left(\frac{\pi}{2}-\theta\right)=\left|m_{g^{-1}}\right|, \operatorname{tg}(\beta)=\left|m_{f}\right|, \operatorname{tg}(\alpha)=\left|m_{f_{s}}\right|
$$

So by plugging in (15) into inequality (18) we will get:

$$
\operatorname{tg}(\beta)-(1-\eta)\left[\left|m_{g^{-1}}\right|+\left|m_{f}\right|\right]<\left|m_{g^{-1}}\right|
$$

Solving (19) can give us a limit for $\eta$ as follows:

$$
\eta<\frac{2 m_{g^{-1}}}{m_{g^{-1}}+m_{f}}
$$

Consequently the optimal value for gain $\eta^{*}$, can be obtained if $\alpha=0$ so the algorithm will converge in one step. Substituting the value of $\alpha$ as zero in (15) will give the optimal value for gain $(\eta)$ :

$$
\eta^{*}=\frac{m_{g^{-1}}}{m_{g^{-1}}+m_{f}}
$$

We have calculated the gain values, $\eta$ and $\eta^{*}$ to guarantee convergence for all typical scenarios depicted in Figure 4-2 which covers the least to most dense possible scenarios in VANET and from low rates to $10 \mathrm{~Hz}$. These values can be observed in Table 4-1. 
Table 4-1 gain( $\eta$ ) values for typical scenarios, with $P_{\max }=25 \mathrm{dBm}$ and $P_{\text {min }}=5 \mathrm{dBm}, U_{\max }=0.8, U_{\min }=0.4$

\begin{tabular}{|c|c|c|}
\hline Scenario & $\begin{array}{c}\eta \text { upper } \\
\text { bound }\end{array}$ & $\eta$ best value \\
\hline$\rho=0.1$, rate $=2.5,10$ & arbitrary & arbitrary \\
\hline$\rho=0.05$, rate $=5$ & 1 & 0.5 \\
\hline$\rho=0.04$, rate $=5$ & 0.94 & 0.47 \\
\hline$\rho=0.03$, rate $=5$ & 0.94 & 0.47 \\
\hline$\rho=0.05$, rate $=2.5$ & 1.07 & 0.53 \\
\hline$\rho=0.04$, rate $=2.5$ & 1.1 & 0.55 \\
\hline$\rho=0.02$, rate $=5$ & 0.97 & 0.48 \\
\hline$\rho=0.03$, rate $=2.5$ & 1.1 & 0.55 \\
\hline
\end{tabular}

To see how the gain affects the convergence speed of the algorithm, we used MATLAB simulations as seen in Figure 4-5. The result for one scenario with $\rho=0.04$ and rate $=5$ can be observed in Figure 4-5. As it can be observed in that figure for assuring convergence the upper bound for $\eta$ should be satisfied and as we choose a value closer to the optimal $\eta$ the convergence will happen faster. We later present NS3 based simulations for evaluation of the findings in this section. The MATLAB tests were done to study the effects of different possible values for the gain $(\eta)$. In CVSS fast convergence matters because road density is subject to change and it can be seen in Table 4-1 that the best values for $\eta$ are close for different scenarios since the behavior of typical scenarios were observed to be very similar. 

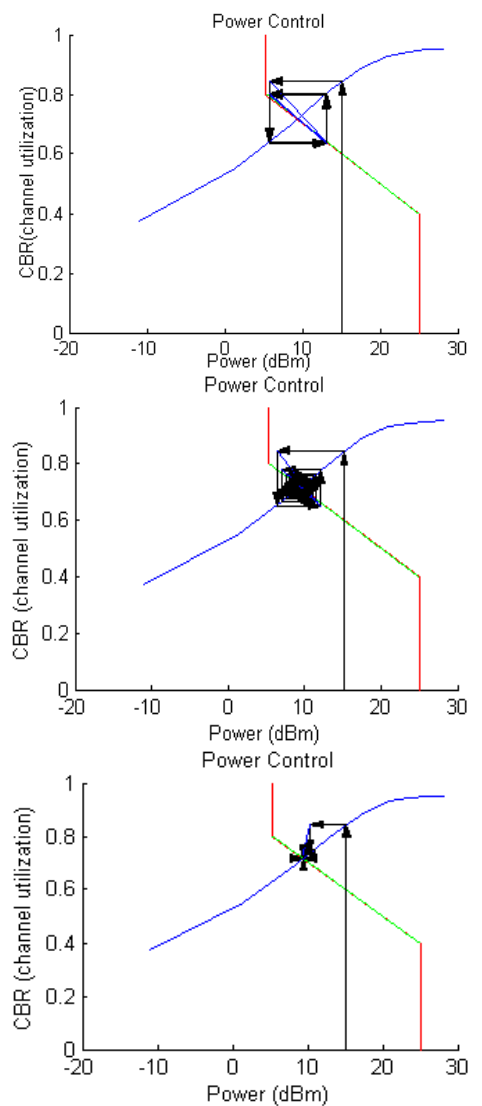

Figure 4-5 matlab simulation for $\rho=0.04$ and rate $=5$ top) SUPRA divergence with $\eta=0.96$ middle) SUPRA convergence with $\eta=0.9$ Bottom) SUPRA fast convergence with $\boldsymbol{\eta}=\mathbf{0 . 5}$

In next part the result of SUPRA for static and realistic highway scenarios will be presented, the stability in time and fairness is observed throughout the results. However there is very slight unfairness issue at edge nodes, which is resolved by mechanisms explained in chapter 5 on top of SUPRA. 


\subsection{Result of SUPRA on Static and Realistic Scenarios}

The value for parameter $\eta$ has been chosen based on the analysis in previous section. This analysis beside information obtained from Table $4-1$ helped us to choose $\eta=0.5$ for our NS3 simulations. Two sets of simulation scenarios are being studied in this chapter: 1) static highway scenarios 2) realistic highway scenarios. The result for static highway scenarios with density of $\rho=0.05$ and $\rho=0.03$ are chosen for static cases since our previous studies showed that these scenarios which are crossing the operating area of the algorithm are the most delicate ones. For very dense and very sparse scenarios adaptation usually consists of less number of iteration and mostly sticks to minimum or maximum of transmission power respectively; therefore, these densities are studied to make sure the system will stabilize. The result for these scenarios can be observed in Figure 4-6 and Figure 4-7. Robustness and fast convergence can be observed in results which would agree with our previous analysis. 

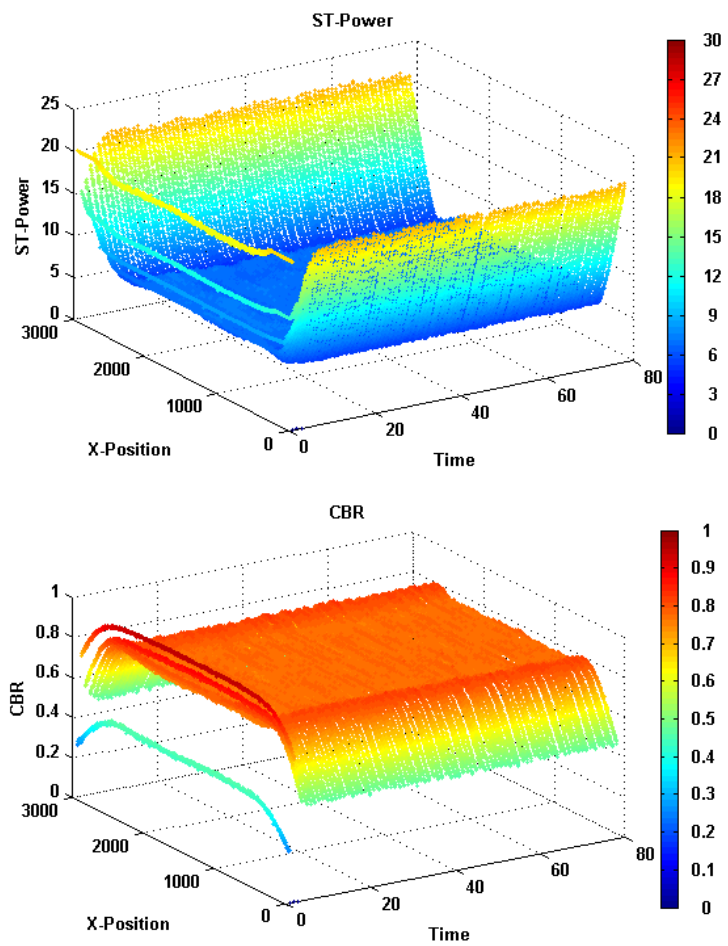

Figure 4-6 SUPRA algorithm result $\eta=0.5$, for $\rho=0.05$ and rate $=5 \mathrm{~Hz}$
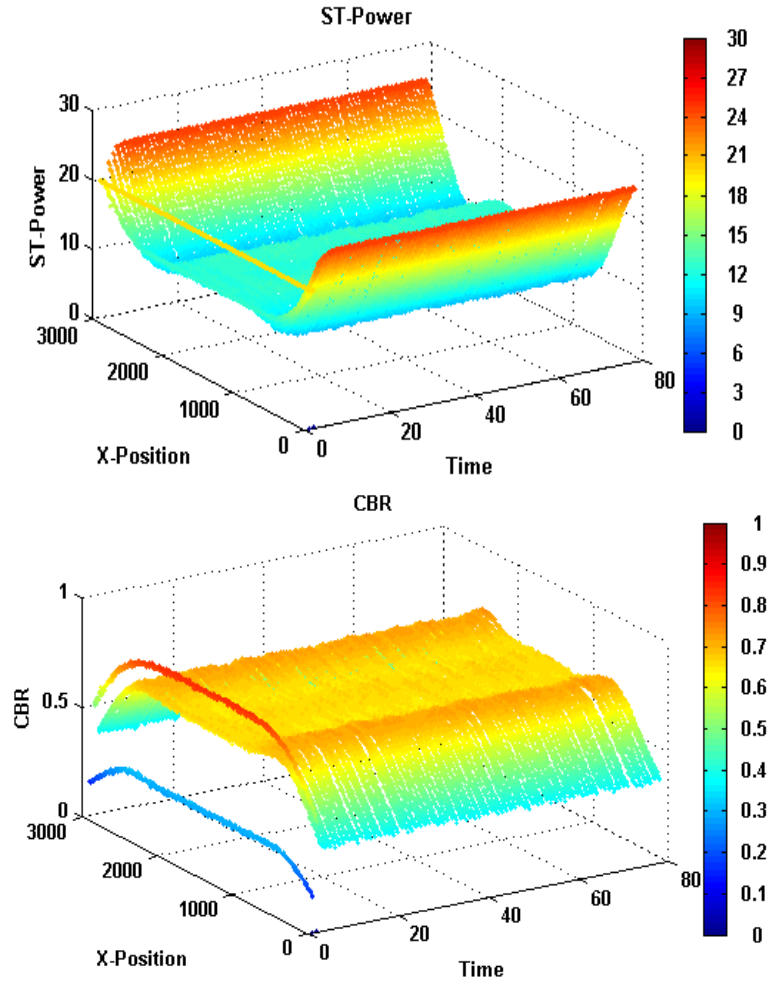

Figure 4-7 SUPRA algorithm, $\eta=0.5$, scenario $\rho=0.03$ and rate $=5 \mathrm{~Hz}$ 
The next set of simulation scenarios consist of 2 realistic scenarios free flow and low speed. The description of these scenarios is the same as chapter 3.4.1 and the maximum speed for each scenario could be observed in Table 3-2. The only difference here is usage of guard message which is $1 \mathrm{~Hz}$ message with maximum power. The intention of using the guard message is for fairness enhancement which will be addressed in next chapter.
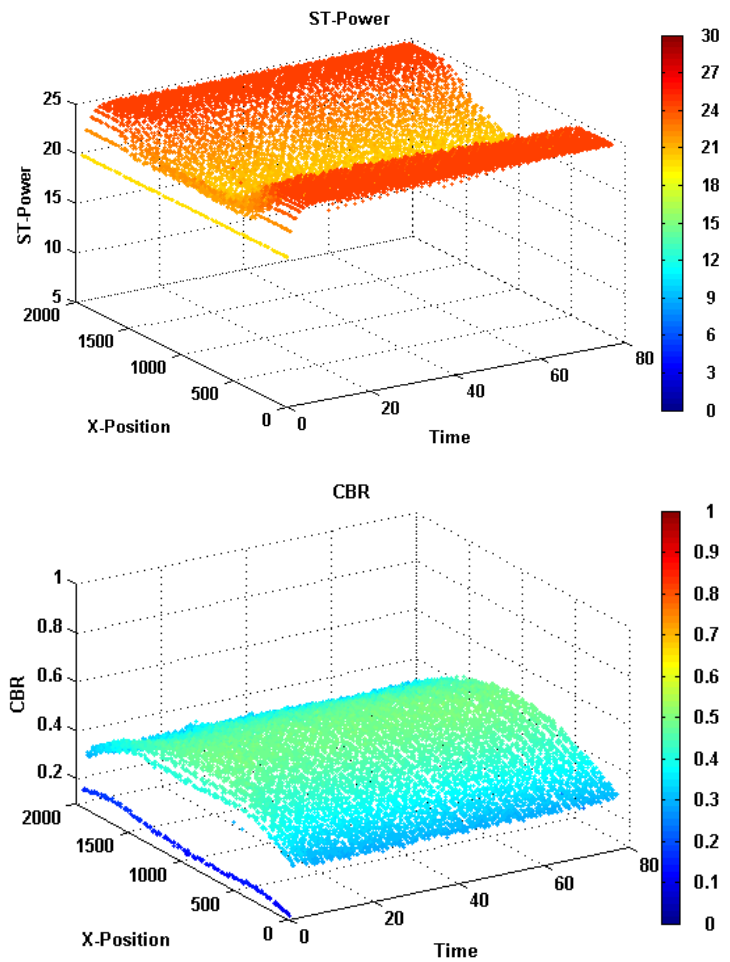

Figure 4-8 SUPRA algorithm result $\eta=0.5$, for realistic scenario (free flow) rate $=5 \mathrm{~Hz}$

The result for these scenarios showed stability and fairness Figure 4-8, Figure 4-9. There is a slight ripple in Figure 4-9 for low speed scenario which can be ignored and will not get worse; however by using fairness enhancement defined in next chapter even this minor ripple will fade away. 

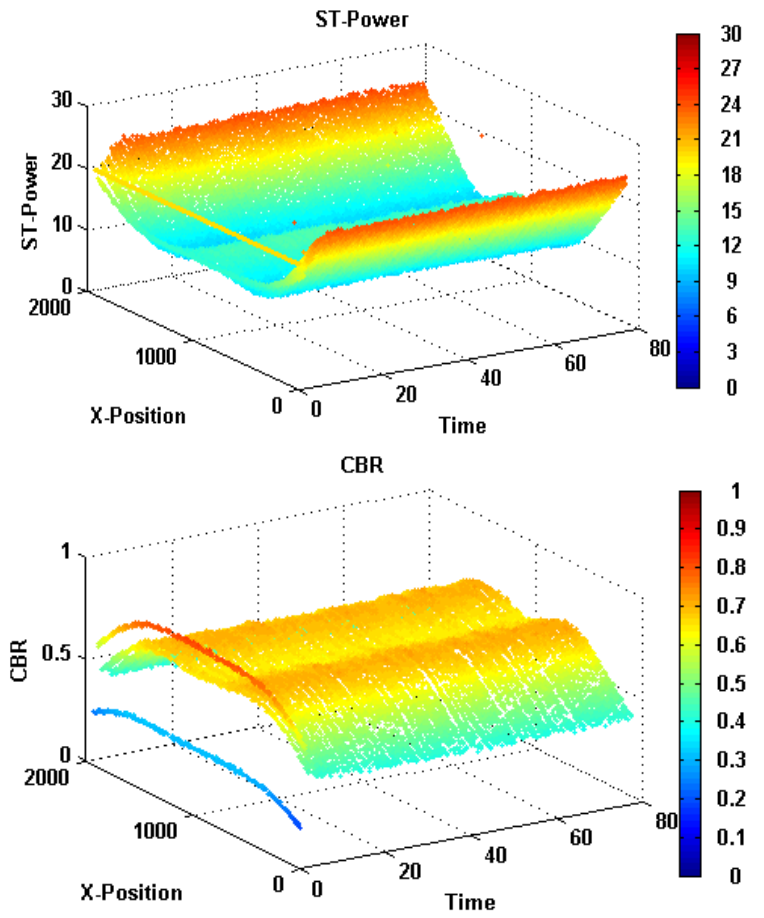

Figure 4-9 SUPRA algorithm result $\boldsymbol{\eta}=0.5$, for realistic scenario (low speed) rate $=5 \mathrm{~Hz}$ 


\section{Chapter 5 : FAIRNESS ASSURANCE FOR POWER CONTROL}

Fairness in accessing the channel is another issue that needs to be studied for any congestion control scheme. The idea is to fairly distribute the burden of congestion control. We define fairness as a spatio-temporal property as follows: nodes which experience the same road density (number of vehicles in a certain space span along the road, e.g., $D_{G}$ meter radius) and have the same average transmission rate should use the same communication range or power. In other words, all nodes should have the same share of the channel. In more crowded situations all nodes should reduce their use of the channel, and not only some select group of nodes. Fairness can also be expressed as:

nodes with the same density should put, on average, the same load on the network; meaning that the product of rate and range, $R \times D$, should be the same for all nodes that are in an area with the same road density.

When power or rate control algorithms based on local measurement of CBR are used, certain unfair situations may be possible. We had done a preliminary study of the LMRC and GRC algorithms in our previous works [18][21], and had particularly noted the issue with the GRC algorithm. The LMRC algorithm was shown to be more robust to unfairness. In this section we provide a more comprehensive study of the LMRC, GRC and the SUPRA algorithm proposed in this paper, and propose an enhancement to how CBR measurement is used in congestion control. 
The unfair situation can be described as a situation in which on a road with the same road density and rate of transmission, a group of cars experience a low CBR while another group at a farther distance measures a high CBR. The group with low CBR will increase its power, further increasing the CBR of the second group. The higher CBR will drive the second group to use a lower power, further decreasing the CBR of the first group. The positive feedback situation causes very different power assignments while road density and transmission rates are the same for all nodes. Resolving the situation requires breaking the positive feedback loop as explained later in this section. Fortunately, this unfair situation is not easy to generate and cannot be observed in many typical situations. Nevertheless, one scenario where we observe the unfairness is when there is a very sharp change in road density, for example as in Figure 5-1, where cars don't exist on part of the road and then a high density of cars appears, such as the edge nodes situation. The edge nodes naturally sense lower value of CBR since they have a lower density of less than half of the road density $(\rho / 2)$ for the first $D_{G}$ meters (if a radius of $D_{G}$ is considered for density measurement); this can be observed in Figure 5-1. Therefore, based on the dynamics of the control schemes used, the edge nodes set their range of transmission to a higher value than the nodes in the middle of the road. This situation is fair for the first $D_{G}$ meters or so because of different densities; however, as time goes on, with some algorithms such as GRC an unfair situation gradually develops farther from the edges, and a low-high rippled effect can be seen (Figure 5-2) along the road where nodes with the same density and rate observe high and low CBRs and set their power accordingly, which is unfair. 


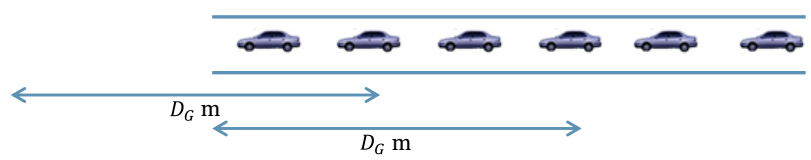

Figure 5-1.The edge nodes density

This situation is more severe for GRC algorithm since it targets a specific CBR value (around 0.65) which happens at a value close to maximum range of transmission; therefore, the unfairness progresses until max power is assigned to these nodes with hypothetical lower density and the other ones converge to a value that gives them a CBR of 0.65. However LMRC and the proposed algorithm SUPRA work with a range of acceptable CBR values, thus assign a gradually decreasing power to nodes around the edge and avoid the unfair situation above, except for minor unfair situation like in Figure 4-9. In this section we examine and propose a few methods for overcoming this unfairness issue for LMRC and SUPRA algorithms. Since GRC is not currently considered for adaptation by the industry we will leave the problem open for later on research. 

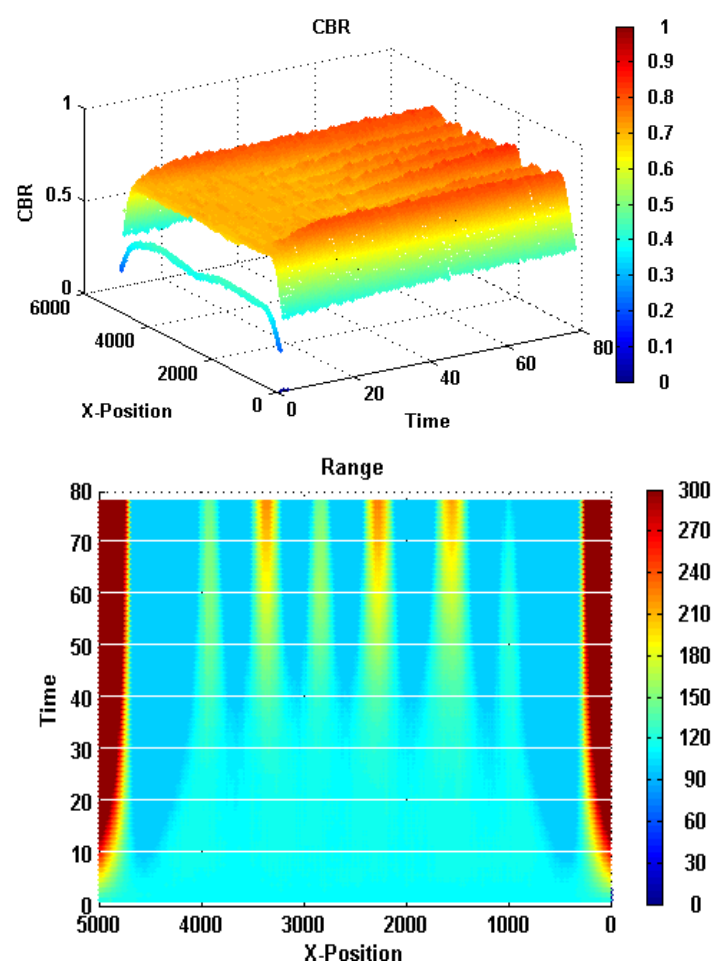

Figure 5-2 GRC algorithm for scenario $\rho=0.05$ and rate $=5 \mathrm{~Hz}$ with gain $=\mathbf{5 0}$

We determined the main cause of unfairness to be the fact that control schemes use local measurements of CBR, while their power will have impact on far nodes. CBR is intrinsically a feedback measure that includes effects of nodes up to one hop far from the sensing node. Nevertheless, when nodes from one group use lower power than another, the effect on CBR will be unbalanced and the distributed nature of the local CBR measurement will be incomplete. To address this issue, we extend the perspective of nodes in sensing CBR using a distributed method. In this method in order to solve the issue of unfairness in space, we have introduced a distributed mechanism. In this method every node considers all measurements of CBR by its neighbors as well as its own locally measured CBR. These distributed measurements of CBR could be aggregated using 
different methods. We have used Averaging and Min-Max method which will be introduced next.

\subsection{Using Averaging Method}

In order to solve the issue of unfairness in space as explained briefly before, we have introduced a distributed mechanism. In this method every node considers all measurements of CBR by its neighbors as well as its own locally measured CBR. Here we will introduce an aggregation method named "Averaging". In this method every node will send the sensed CBR of its channel along with the safety packets to its neighbors. This value is added to the safety message and no extra protocol is required and no more overhead is forced. Using neighboring node's CBR will help each node to have a wider picture of the network, which can be helpful in resolving the unfairness issue. We chose to use averaging over all heard CBR values from neighbors. This method worked almost perfectly for the fixed scenarios. The result for one of the fixed scenarios which had unfairness issue before using averaging is shown in Figure 3-8. The same case is depicted in Figure 5-3 and shows that the unfairness issue is resolved by using the averaging method.

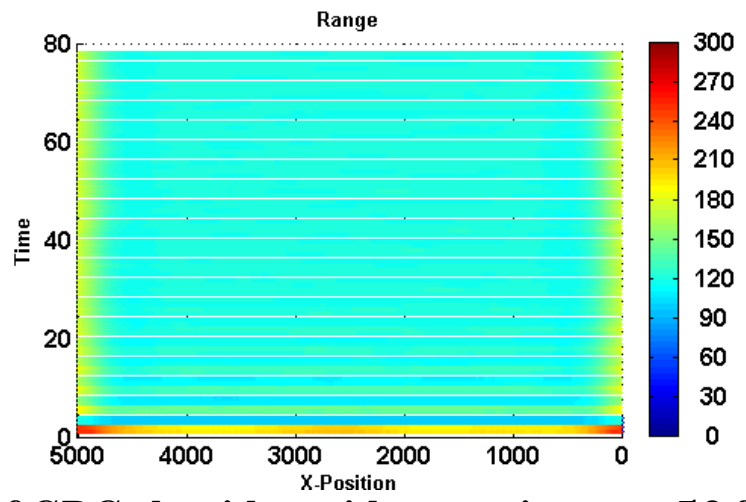

Figure 5-3 Result of GRC algorithm with averaging, $\eta=50$ for case $\rho=0.05$ and rate $=5$

It was shown here that a distributed measurement of CBR feedback will allow more fairness in channel access and transmission power assignment. In Figure 3-15 the results of 
running GRC with distributed CBR measurement are shown for the congested case with $\rho=0.2$. As it can be observed in this case, comparing with the pure GRC application in Figure 3-12, edge nodes are not selecting higher range of transmission and the range values assigned to all vehicles are fair. We can see in Figure 3-12 that the edge nodes range is increasing in time since the density at those positions is much smaller. However by using averaging, these nodes will receive the high CBR being sensed at their neighbors, which is piggybacked in safety messages received. The edge nodes consider these CBR values and will have a thorough picture of the whole network and adapt their range accordingly. Therefore, by this method the range would be chosen in a more distributed fashion rather than local. CBR measurement is a limited feedback of the channel and in cases of large networks with different behavior of nodes this local CBR value can mislead the control function. Unfairness is an example of this phenomenon, in which locally measured CBR could be very high as a result of neighboring nodes setting their range of transmission to a high value (sometimes max range); in such cases obeying the locally measured CBR can lead to an unfair situation which is not desirable in CVSS systems. By utilizing distributed measurements of CBR we will have a more robust picture of the network and will be sure to do the adaptation in a way that each node has a fair share of the channel. This method results in fair range adaptation for all the vehicles along the road and was used on top of GRC algorithm throughout this study.

The next scenario to study is the mixed scenario. Looking at the results in Figure 3-16, the edge effect does not show much since the edge nodes naturally have the low density and consequently they will set their transmission range to maximum. The time stability and apace fairness is observed. 
The problem with averaging method is that there are cases in which the average CBR is stabilized while there is still unfairness. It means that there can be some case in which we have extremely high $\mathrm{CBR}$ and extremely low CBR which are distributed such that their average is around stability point and therefore the averaging method can be misleading. This was the motivation to look for another method in which we are mostly looking at individual CBR measurements combined with a resource allocation mechanism named Min-Max approach. This method is presented in next section.

\subsection{Using Min-Max Method}

The improved method of computing a feedback measure as discussed is based on having sensed local CBR piggybacked on CVS messages to the neighboring nodes. The broadcast is done up to one adjacent hop (up to $D_{G}$ reached by guard messages). The overhead is negligible as the value of CBR can be reported in only a few bits. At each node the maximum value of all these received CBRs and the locally sensed CBR will be considered and used for the control purpose. Due to the dynamics of the control schemes, higher values of CBR will lead to lower ranges (powers) of transmission; this is in accordance with the fact that control function is a decreasing function of CBR. The algorithm can be summarized below; a summary proof of why such a measure will provide fairness is provided next. 


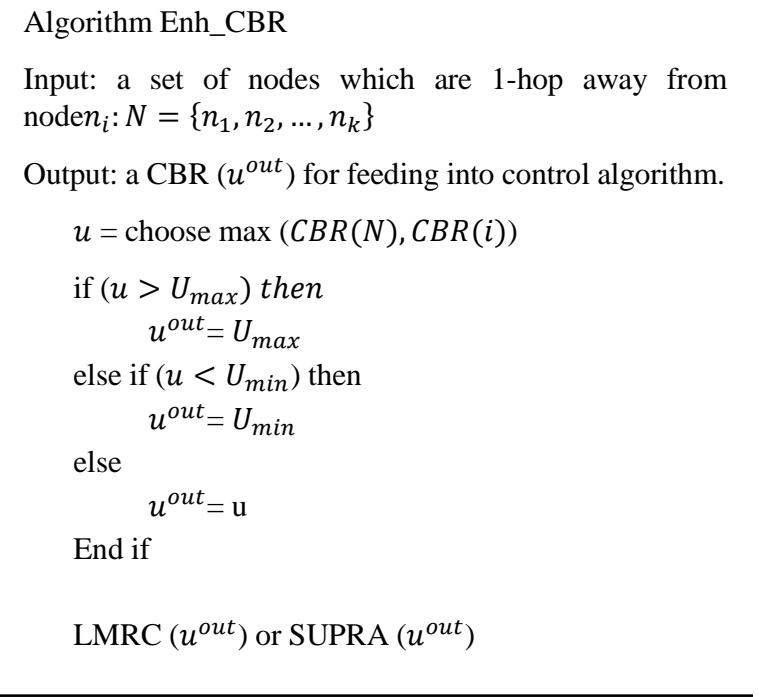

\subsection{Analysis of CBR Enhancement Algorithm}

The LMRC and proposed power control algorithm, SUPRA, work with a measurement of CBR. So for any two nodes $n_{i}$ and $n_{j}$, which have the same road density and rate (thus observing the same network characteristic curve), if $C B R_{i}=C B R_{j}$ then both LMRC and SUPRA will result in the same power for both nodes $\left(P_{k+1}\right)$ in a few iterations (as was shown in the stability section). Now we will prove that even if $C B R_{i} \neq C B R_{j}$, by using the enhancement proposed in this section, the situation will be resolved in few iterations of the enhancement algorithm.

Suppose we have the situation as depicted in Figure 5-4 and all nodes experience the same density. nodes $\mathrm{u}, \mathrm{v}$ and $\mathrm{w}$ are sensing a low CBR therefore setting their power to a high value causing nodes $\mathrm{q}$, and $\mathrm{z}$ to sense higher CBRs and setting their power to low values. This case is seen in simulation runs. In this scenario nodes $\mathrm{u}, \mathrm{v}$, and $\mathrm{w}$ contribute to the CBR at $\mathrm{q}$ and $\mathrm{z}$, while $\mathrm{q}$ and $\mathrm{z}$ use very low power due to the higher CBR (interposed by other nodes) and will not add to the CBR in u,v,w. The result is the situation in which 
nodes $\mathrm{u}, \mathrm{v}, \mathrm{w}$ end up with high power and low CBR, while nodes $\mathrm{q}$ and $\mathrm{z}$ use low power due to high CBR. Assuming this situation, if we introduce the enhancement algorithm using maximum reported CBR, the unfairness will resolve as explained next.

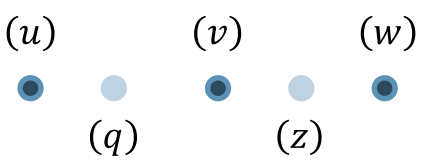

Figure 5-4 Nodes $q$ and $z$ use lower power due to higher local CBR (without the enhanced algorithm)

The CBR enhancement algorithm will allow all nodes in $D_{G}$ range of each other to share the sensed $\mathrm{CBR}$. The use of a mechanism such as maximum CBR, will ensure that nodes that are hearing the same set of CBR values will pick a single CBR value (the maximum) and become synchronized in their next power setting decision. For the example of Figure 5-4, if $\mathrm{D}_{\mathrm{G}}$ is such that only 2 neighbors are covered, then the nodes $\mathrm{u}, \mathrm{v}$ and $\mathrm{w}$ that have low CBR will follow one of the nodes q or $\mathrm{z}$ and will lower their power, which will cause lower CBR for $\mathrm{q}$ and $\mathrm{z}$, and in few iterations this trends will cause equal CBR for all these nodes.

In a memory-less algorithm like LMRC, this means that the next value of power (or range) would be the same for all nodes sharing the same CBR set. For stateful algorithms like SUPRA, the synchronization takes few steps since the initial choices of power will dictate the new value. To see why the nodes eventually converge to the same power setting, consider the fact that the algorithm for SUPRA can be written as : 


$$
\begin{gathered}
P_{k+1}=P_{k}+\eta \times\left(f\left(U_{\text {max }}^{k}\right)-P_{k}\right)=P_{k}(1-\eta)+\eta\left(f\left(U_{\max }^{k}\right)\right) \\
=P_{k-1}(1-\eta)^{2}+\eta(1-\eta)\left(f\left(U_{\max }^{k-1}\right)\right)+\eta\left(f\left(U_{\max }^{k}\right)\right) \\
=P_{k-j}(1-\eta)^{j+1}+(1-\eta)^{j} \eta f\left(U_{\max }^{k-j}\right)+H\left(j, U_{\max }^{k}, U_{\max }^{k-1} \ldots U_{\max }^{k-j}\right)
\end{gathered}
$$

where $\mathrm{H}$ abstract the summation of previous fractions of Umax values since iteration $k-j$ is not directly correlated with the nodes choice of power at iteration $k-j$. As it can be seen the effect of $P_{k-j}$ and $f\left(U_{\max }^{k-j}\right)$ is faded over iterations with factors $(1-\eta)^{j+1}$ and $\eta(1-\eta)^{j}$. With increasing iteration $\mathrm{j}$, the effect of the initial values fade and the nodes behave similarly. With nodes synchronized in their power/range setting, the algorithm progresses and converges under the stability conditions described in chapter 4.

An illustration of the unfair situation can be visualized in Figure 5-5, which shows that without the enhancement and in the unfair situation nodes with high and low CBR will observe false network characteristic curves that are due to the unbalance in power assignments. Note that network characteristic curves are derived assuming similar behavior from all nodes, which is not the case in unfair situations. The sharing of CBR and choosing a single value will cause the nodes to behave similarly and therefore the observed characteristic curve will be the actual one and will guide the convergence in time.

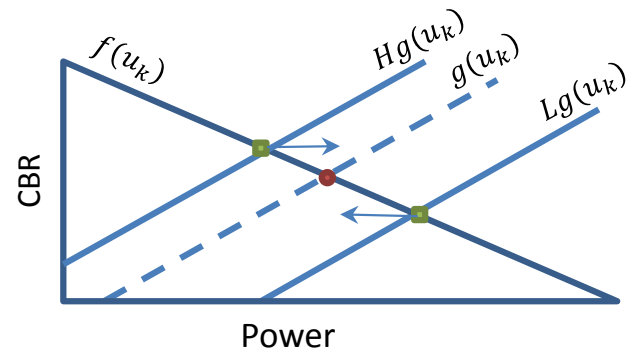

Figure 5-5 In an unfair situation, false network characteristic curves $\mathrm{Hg}$ and $\mathrm{Lg}$ are observed by nodes seeing unbalanced high and low CBRs, resulting in false and unfair stability point (square); the real network characteristic curve $\mathrm{g}$ and its stability point (circle) will result when nodes are synchronized using the enhanced algorithm. 
To get more insight into how each iteration of the algorithm with shared CBR works, the following table shows different iterations following a hypothetical unfair situation.

Table 5-1 Hypothetical unfairness resolved by using fairness enhancement

\begin{tabular}{|c|c|c|c|c|c|}
\hline Nodes & $\mathrm{u}$ & $\mathrm{q}$ & $\mathrm{v}$ & $\mathrm{z}$ & $\mathrm{w}$ \\
\hline$U_{k}$ & $C B R_{u}$ & $C B R_{q}$ & $C B R_{v}$ & $C B R_{z}$ & $C B R_{w}$ \\
\hline$P_{k}$ & $P_{u}$ & $P_{q}$ & $P_{v}$ & $P_{z}$ & $P_{w}$ \\
\hline$U_{k+1}$ & $C B R_{v}$ & $C B R_{v}$ & $C B R_{v}$ & $C B R_{v}$ & $C B R_{v}$ \\
\hline$P_{k+1}$ & $P_{u} \downarrow$ & $P_{q} \downarrow$ & $P_{v}$ & $P_{z} \downarrow$ & $P_{w} \downarrow$ \\
\hline$U_{k+2}$ & $C B R_{u} \downarrow$ & $C B R_{q} \downarrow$ & $C B R_{v} \downarrow$ & $C B R_{z} \downarrow$ & $C B R_{w} \downarrow$ \\
\hline$U_{k+2}$ & $C B R_{v}$ & $C B R_{v}$ & $C B R_{v}$ & $C B R_{v}$ & $C B R_{v}$ \\
\hline
\end{tabular}

To verify the effect of the enhancement, we have run the simulation and compared the result with the original method. The enhancement improves many of earlier presented results in terms of providing a fairer channel to all the vehicles. For example results in Figure 5-6 show the same scenario as in Figure 4-9, but with the CBR enhancement. A considerable improvement can be observed and the slight ripple in the result is faded away. 

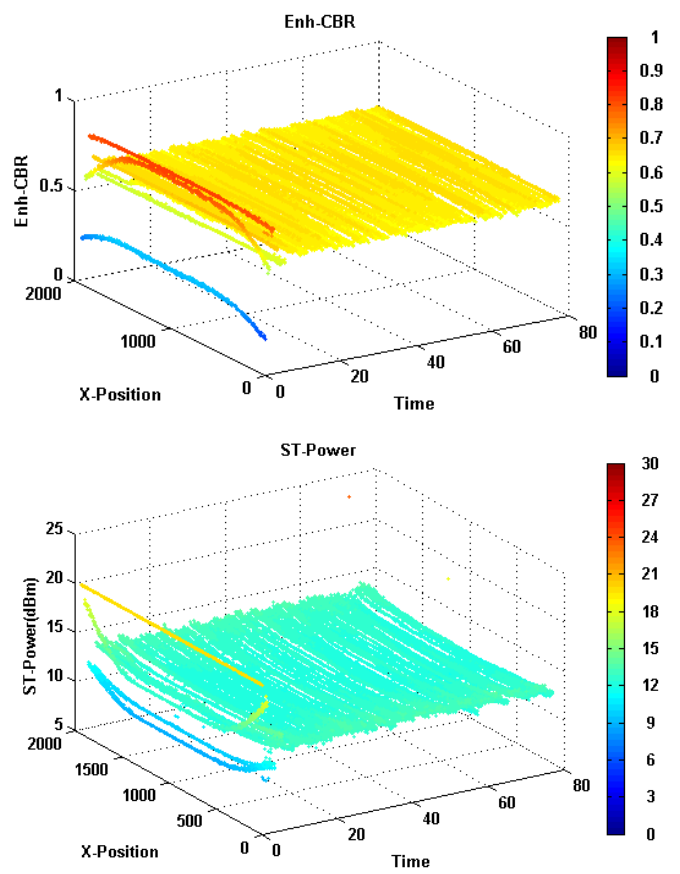

Figure 5-6 SUPRA algorithm, $\eta=0.5$ and Enhancement applied on top of SUPRA, realistic scenario (low speed) rate $=5$
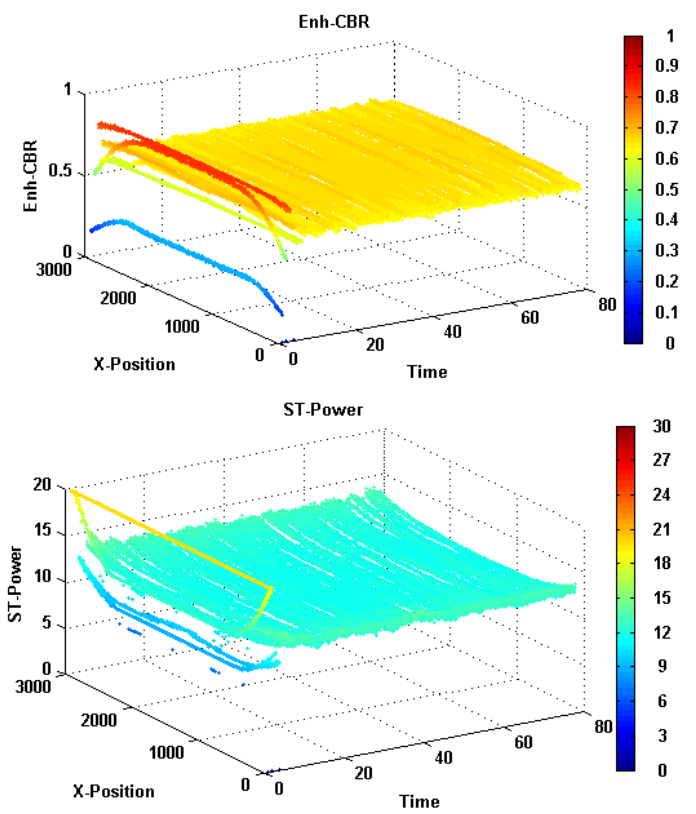

Figure 5-7 SUPRA algorithm, $\eta=0.5$ and Enhancement applied on top of SUPRA, scenario $\rho=0.03$ and rate $=5 \mathrm{~Hz}$ 
Another scenario studied here is $\rho=0.03$, the result can be observed in Figure 5-7. This result could be compared against Figure 4-7. The minor variation in power assignment in vanished by using the fairness enhancement on top of SUPRA algorithm.

To better see how the algorithm resolves unfairness, we manually created unfair power assignments and then observed that the enhanced algorithm completely resolved the situation as is seen in Figure 5-8 and Figure 5-9.
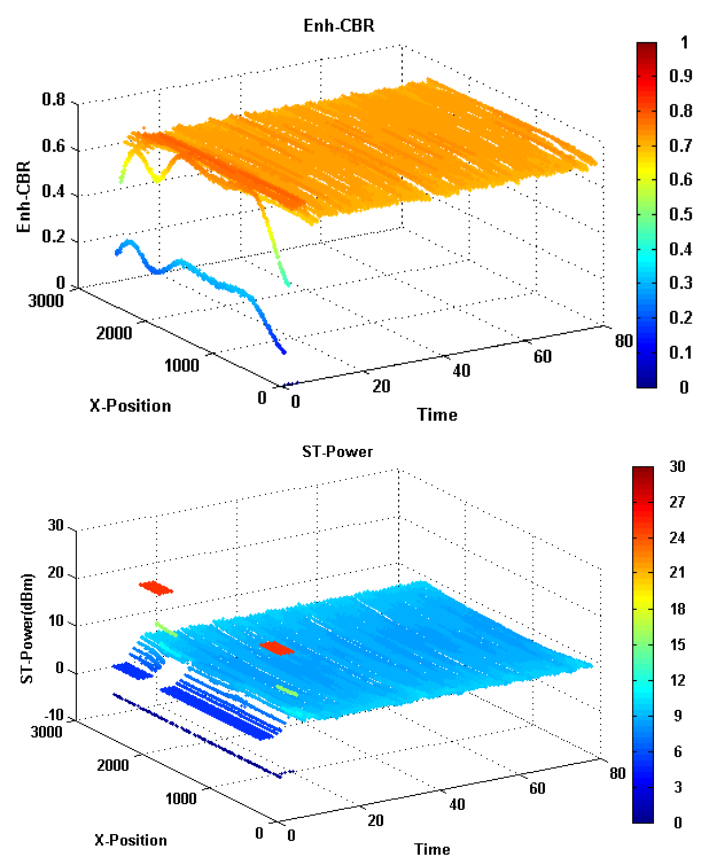

Figure 5-8 SUPRA algorithm, $\eta=0.5$ and Enhancement applied on top of SUPRA, scenario $\rho=0.04$ and rate $=5 \mathrm{~Hz}$ with unfairness build up

A noticeable progress in fairness can be seen in the result after applying fairness enhancement in Figure 5-8. The slight edge effect and ripple in the first 500meter of each side of the edges is resolved after using the fairness enhancement. It should be noted that the unfairness build up is resolved by the algorithm mechanism and the observed 
unfairness is a result of edge nodes which could get alleviated considerably by utilizing the fairness enhancement algorithm on top of SUPRA.
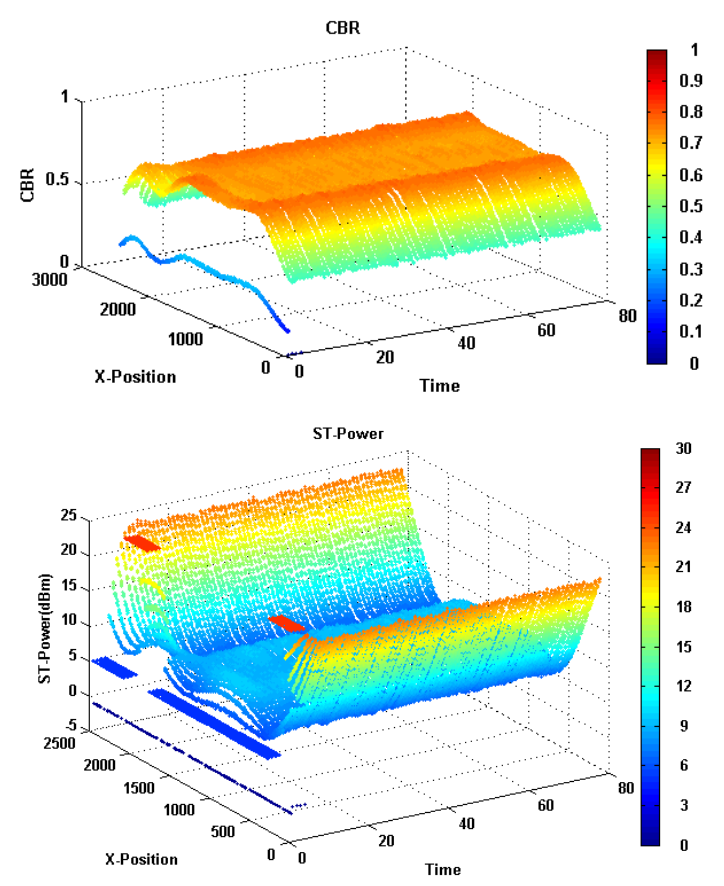

Figure 5-9 SUPRA algorithm, $\eta=0.5$ and No Enhancement, scenario $\rho=0.04$ and rate $=\mathbf{5 H z}$ with unfairness build up

SUPRA algorithm with fairness enhancement is proved to be stable and fair for most typical road scenarios and average rates of transmission. In contrast with LMRC this algorithm does not impose limitations on minimum and maximum of CBR nor Power. The direct power assignment of SUPRA algorithm will prevent inconsistencies in mapping the range to power for different environments and different vehicles. This algorithm generally can be considered as a reliable and safe congestion control mechanism for Cooperative Vehicular Safety Systems and guarantee updated information of all the vehicles thru the network to assure safety to all the drivers. This is possible due to fair shared channel and control over the power of transmission based on channel situation specified by the channel feedback (CBR). 


\section{Chapter 6 : CONCLUSION AND FUTURE WORK}

Cooperative vehicle safety systems are the most important and challenging application of VANETs. These systems need to be robust enough in case of large scale implementation. The size of these networks is subject to rapid increase especially in 10-12 lane highways existing today. In case of road congestion on these huge highways the number of vehicles in range could grow extremely large; therefore, the control system designed for CVSS should be robust and able to operate at a certain level of assurance in case of large scale networks. To achieve scalability, there have been considerable amount of effort from researchers and industry to design a scalable and reliable system for congestion control. Most of these mechanisms are based on the most effective levers of the system: 1) rate of transmission 2) range/power of transmission. The adaptation of these levers should be designed based on a feedback of the system (plant) which is commonly CBR in most recent studies. In this work we have examined the dynamic behavior of two of existing algorithms named: GRC and LMRC to verify their stability and fairness property. Certain restriction should be considered in order to satisfy convergence conditions especially for LMRC algorithm. These restrictions can be met most of the time however they could be restraining in some cases. Additionally fairness issue was investigated for GRC and LMRC algorithms. Fairness issue is very important in CVSS since it could cause starvation to some vehicles on the road and put them in danger of not being heard from other vehicles. This issue was addressed using a distributed measurement of CBR instead of limited local 
CBR. This would make a wider perspective of the network to each node and therefore result in much robust and fair result.

CVSS application in urban environments is an important subject. One main issue in urban area is intersection collision warning. The limited line of sight at intersection makes it very important to have robust communication between vehicles. In this study intersection scenarios were studied to assure algorithms stability and fairness in both highway and intersection situations. This work has been verified through realistic scenarios obtained from SUMO urban simulator.

The possible directions for future studies of this work are suggested as follows. One important study is to examine the suggested power control SUPRA simultaneously with rate control. It has been shown in [18] that rate of transmission is a function of driver maneuver behavior. Dangerous driving patterns need higher rate of update while a constant speed movement without lane change does not need very frequent information update in order to help other vehicles estimate the next position of the vehicle. This idea can be combined with robust range/power control mechanism introduced in this work to improve the congestion control mechanism. Involving rate adaptation on top of range/power control can cause unfairness as well; therefore, studying unfairness for the joint adaptation can be another direction for future research. The unfairness issue study for joint adaptation could be more complicated and need a new measure of fairness since both rate of transmission and density of vehicles are subject to change while in this study rate was assumed to be the average rate. 


\section{References}

[1] Vehicle Safety Communications Consortium (VSCC), "Vehicle Safety Communications Project, Task 3 Final Report: Identify Intelligent Vehicle Safety Applications Enabled by DSRC," 2005.

[2] IEEE 802.11 WG, Part 11: Wireless LAN Medium Access Control (MAC) and Physical Layer (PHY) Specifications, IEEE, Aug. 1999.

[3] Wireless Access in Vehicular Environment (WAVE) in Standard 802.11, Specific Requirements: IEEE 802.11p/D1.0, Feb. 2006.

[4] IEEE 1609.3 Wireless Access in Vehicular Environment: Networking Services, 2007.

[5] R. Sengupta, S. Rezaei, S. E. Shladover, J. A. Misener, S. Dickey, H. Krishnan, "Cooperative Collision Warning Systems: Concept Definition and Experimental Implementation," Journal of Intelligent Transportation Systems, vol.11,No.3, pp. 143$155,2007$.

[6] G.Bianchi,"Performance Analysis of the IEEE 802.11 Distributed Coordination Function,” IEEE J. Selected Areas Comm, vol.18, no.3, pp.535-547,Mar.2000.

[7] X. Yang, "IEEE 802.11e wireless LAN for quality of service," in Proc. IEEE Wireless Commun. Netw. Conf., 2003, pp. 1291-1296.

[8] G. R. Cantieni, Q. Ni, C. Barakat, and T. Turletti, "Performance analysis under finite load and improvements for multirate 802.11," Comput. Commun., vol. 28, no. 10, pp. 1095-1109, Jun. 2005.

[9] M. S. Bouassida, M. Shawky, “A Cooperative and Fully-Distributed Congestion Control Approach within VANETs," Intelligent Transport Systems Telecommunications,(ITST), 2009 9th International Conference on. IEEE, 2009.

[10] X. Guan, R. Sengupta,h. Krishnan, F. Bai, “A Feedback-Based Power Control Algorithm Design for VANET," 2007 Mobile Networking for Vehicular Environments. IEEE, 2007.

[11] M. Sepulcre, J. Gozalvez, J. Harri, H. Harstenstein, "Contexual Communication Congestion Control for Cooperative Vehicular Networks," Wireless Communications, IEEE Transactions on 10.2 (2011): 385-389. 
[12] Y. Zang, L. Stibor, X. Cheng, H. Reumerman, A. Paruzel, A. Barroso, "Congestion Control in Wireless Networks for Vehicular Safety Applications," Proceedings of the 8th European Wireless Conference. Vol. 7. 2007.

[13] Y.P. Fallah, C.L. Huang, R. Sengupta, H. Krishnan, "Congestion Control Based on Channel Occupancy in Vehicular Broadcast Networks", proc. IEEE Vehicular Tech. Conf VTC Fall 2010.

[14] C. L. Huang, Y. P. Fallah, R. Sengupta, H. Krishnan, "Information Dissemination Control for Cooperative Active Safety Applications in Vehicular Ad-Hoc Networks," Proc. of IEEE Globecom 2009

[15] T. Tielert, D. Jiang, Q. Chen, L. Delgrossi, H. Hartenstein, "Design Methodology and Evaluation of Rate Adaption Based Congestion Control for Vehicle Safety Communications”, Proc of IEEE Vehicular Networking Conference (VNC),2011

[16] R. Baldessari, D. Scanferla, L. Le, W. Zhang, A. Festag, "Joining Forces for VANETs: A Combined Transmit Power and Rate Control Algorithm," th International Workshop on Intelligent Transportation (WIT). 2010.

[17] M. Torrent-Moreno, J. Mittag, P. Santi, H. Hartenstein, "Vehicle-to-Vehicle Communication: Fair Transmit Power Control for Safety-Critical Information," IEEE Trans. on Vehicular Technology, Vol 58, Issue 7, pp. 3684 - 3703, Jan 2009

[18] C. L. Huang,Y. P. Fallah, R. Sengupta, H. Krishnan, "Intervehicle Transmission Rate Control for Cooperative Active Safety System", IEEE Trans on Intelligent Transportaion Systems, Vol. 12 NO. 3. September.

[19] S. Rezaei, R. Sengupta, H. Krishnan, X. Guan, R. Bhatia, "Tracking the Position of Neighboring Vehicles Using Wireless Communications," Elsevier Journal Transportation Research Part C: Emerging Technologies, Special Issue on Vehicular Communication Networks, vol 18, issue 3, pp 335-350, 2010

[20] C. L. Huang, Y. P. Fallah, R. Sengupta, H. Krishnan, "Adaptive InterVehicle Communication Control for Cooperative Safety Systems", IEEE Network, Jan.-Feb. 2010, Vol. 24, Issue 1, pp. 6-13

[21] N. Nasiriani, Y. P. Fallah, H. Krishnan, "Stability Analysis of Congestion Control Schemes in Vehicular Ad-hoc Networks", IEEE 10 ${ }^{\text {th }}$ CCNC , Jan 2013 
[22] Y.P. Fallah, C.L. Huang, R. Sengupta, H. Krishnan, “Analysis of Informaiton Dissemination in Vehicular AdHoc Networks of Cooperative Vehicle Safety Systems", IEEE Trans. on Vehicular Technology, Vol 60, Issue 1, pp. 233-247, Jan 2011.

[23] N. Nasiriani,Y. P. Fallah. "Performance and fairness analysis of range control algorithms in cooperative vehicle safety networks at intersections." Local Computer Networks Workshops (LCN Workshops), 2012 IEEE 37th Conference on. IEEE, 2012.

[24] Y. P. Fallah, CL. Huang, R. Sengupta, H. Krishnan, "Design of Cooperative Vehicle Safety Systems Based on Tight Coupling of Communication, Computing and Physical Vehicle Dynamics", Proc. Of IEEE Int. Conference on Cyber-Physical Systems 2010, ICCPS'10

[25] L. Cheng, B. E. Henty, D. D. Stancil, F. Bai, P. Mudalige, "Mobile Vehicle-toVehicle Narrow-Band Channel Measurement and Characterization of the 5.9 GHz Dedicated Short Range Communication (DSRC) Frequency Band", IEEE J. Sel. Areas in Comm., Vol. 25, NO. 8, Oct 2007. 


\section{Neda Nasiriani}

304-906-1983

nnasiria@mix.wvu.edu

Permanent Address

1431 Saratoga Ave, \#8

Morgantown WV 26505
Campus Address

Engineering Research Building 218, WVU, Morgantown WV 26506

\section{Education}

West Virginia University, Morgantown, WV

Pursuing Master in Electrical Engineering, Current

GPA: $4.0 / 4.0$

Sharif University of Technology, Tehran, Iran

BSc. in Computer Engineering, minor Information

Technology Engineering [top ranked university in

Iran], GPA: 16.64/20.0
January 2012- Present

September 2006-January 2010

\section{Conference Publications}

$\checkmark \quad$ N. Nasiriani, Y. P. Fallah, "Performance and Fairness Analysis of Range Control Algorithms in Cooperative Vehicle Safety Networks at Intersections". IEEE LCN ONMOVE Oct 2012

$\checkmark \quad$ N. Nasiriani, Y. P. Fallah, H. Krishnan, "Stability Analysis of Congestion Control Schemes in Vehicular Ad-hoc Networks". IEEE CCNC Jan 2013, acceptance rate $30 \%$

$\checkmark \quad$ N. Nasiriani, R. Ramachandran,K. Rahimi, Y. P. Fallah, P. Famouri, S. Bossart, K. Dodrill, "An Embedded Communication Network Simulator for Power Systems Simulations in PSCAD”. proc of PES general meeting 2013, acceptance rate: $30 \%$

\section{Journal Publications}

$\checkmark \quad$ Y. P. Fallah, N. Nasiriani, "Stable and Fair Power Control in Vehicule Safety Networks", under review, to appear in IEEE Transactions on Vehicular Technology (TVT)

$\checkmark$ E. Moradi-Pari, N. Nasiriani, Y. P. Fallah, P. Famouri, S. Bossart, K. Dodrill "Design, Modling and Simulation of On-Demand Communication Mechanisms for Cyber-Physical energy Systems", under review, to appear in Transactions on Industrial Informatics

\section{Poster Presentations}

$\checkmark \quad$ E. Moradi-Pari, N. Nasiriani, Y. P. Fallah, P. Famouri, S. Bossart, K. Dodrill “An Embedded Communication Network Simulator for Power Systems Simulations in PSCAD”, Electricc Power Industry Cnference, Nov 2013

\section{Research and Teaching Experience}

$\checkmark \quad$ Research Assistant [January 2013-Present]: Working on Development of the DSRC V2V Scalability Simulator at CPSLab at WVU in collaboration with CAMP(Crash Avoidance Metrics Partnership)

$\checkmark \quad$ Research Assistant [August 2012-Present]: Working on development of communication protocols (DNP3 and IEC 61850) for Grid Technology Cyber- 
Infrastructure in PSCAD at CPSLab at WVU in collaboration with GTC (NETL-RUA Grid Technologies Collaborative)

$\checkmark \quad$ Research Assistant [January 2012-Present]: Working on congestion control algorithms of Vehicular Ad-hoc Networks (VANETs) at CPSLab in WVU

$\checkmark \quad$ Intern at Intelligent Solutions, Inc. [May 2013-Aug 2013]: Working on Development of a software to extract large data, load it to database and visualize geological surfaces using OpenGL

$\checkmark$ Software Engineer [Summer 2010]: Developing an interface in VB6 for the engine of a software provided in DLL format at PEARL (Petroleum Engineering and Analysis Research Laboratory) at WVU

$\checkmark$ Research Assistant [Summer 2009]: Working as a research assistant at Digital Media Laboratory (DML) at Sharif University, on the Wearable Gestural Interface (WGI) project.

$\checkmark$ Teacher Assistant [2009]: Assisting “Operating System” course.

\section{Selected Academic Projects}

$\checkmark$ Communication Interface for PSCAD [Aug 2012-Present]: An implementation of Communication protocols for Grid Technology Cyber-Infrastructure in PSCAD

$\checkmark$ Congestion Control Mechanisms in NS3 [Jan 2011-Present]:Implementing Congestion Control Mechanisms(Range and Power control) for VANETs in NS3

$\checkmark \quad$ Routing On Tehran Map [Google Map API,2009]: Developing an application for showing routes on Tehran city map

$\checkmark$ Personal Dictionary [Symfony,php,2009]: Developing a personal online dictionary

$\checkmark$ Project Management [2009]: Managing a project building a virtual bank

$\checkmark$ Computer Museum Management System [Java- EE and MYSQL, 2009]: Developing an integrated web application for managing a computer museum consisting of planning, accounting, partners, visitors, inventory, and management subsystems.

$\checkmark$ Course Scheduler [Prolog, 2009]: Developing a course scheduler for universities.

$\checkmark$ PintOS [C, 2008]: Developing some basic features of operating systems in PintOS.

$\checkmark$ Web Browser [Java, 2007]: Developing a complete web browser for Windows.

$\checkmark$ Monopoly Game Application [Java, 2007]: Developing Monopoly game for Windows.

\section{Technical Skills}

$\checkmark$ Network Simulators: NS-3, Network programming, socket programming

$\checkmark$ Power System: PSCAD (mostly scripting inside PSCAD)

$\checkmark$ Transportation: SUMO

$\checkmark$ Programming Languages: C/C++, Java SE, Java EE, VB6, Google Map API, FORTRAN

$\checkmark$ Web Technologies: HTML, CSS, PHP, Java Script, JSP, Symfony

$\checkmark$ Database Technologies: MYSQL, PostgreSQL

$\checkmark$ Software Engineering: System Architect(SA), MS Project

$\checkmark$ Assembly Languages: Verilog, 8086 IBM assembly language and programming

$\checkmark$ Operating Systems: MS Windows, Linux, PintOS

$\checkmark$ Data Mining: Weka tools

$\checkmark$ Graphics: OpenGL library

\section{Service}

$\checkmark \quad$ IRanian Student Association(IRASA) [Jan 2011-March 2013]: Serving as an officer for IRASA student organization, web master 
Awards and Honors

$\checkmark \quad$ Iraninan American Academics and Professional Scholarship award [Nov 2013]: 17 out of 93 applicants from all distinguished universities from east

\section{Language}

$\checkmark$ Persian: Native

$\checkmark \quad$ English[Aug 2013]: Fluent Toefl score (112/120) 
